\title{
BIBLIOGRAPHY.
}

\section{PRELIMINARY STATEMENT}

While this bibliography is admittedly not complete, it is thought that not many papers of consequence, published up to 1921, have been overlooked. Many descriptions of antiquated processes in the manufacture of gypsum have been omitted. Many such papers are listed by Von Waldegg (Der Gips, Verlag von T. Thomas, Leipsig, 1906).

Acknowledgment is made of assistance received from manuscript bibliographies of H. J. Brown, and V. G. Marani, as well as published bibliographies shown below. The valuable agricultural bibliography of Olson and St. John and the bibliography of DeKalb on sulphur in agriculture, are reproduced in their entirety. In this same connection a manuscript bibliography of Professor William Crocker has proved helpful.

The following plan has been adopted for this bibliography:

1. General references

2. Bibliographies

3. Descriptions of localities United States (as a whole) by states

- Foreign

Canadian European Australia Asia Africa

4. Geological

5. Chemical and Mineralogical

6. Technical

7. Structural Tests

8. Hydraulic gypsum

9. Gypsum in Portland Cement

10. Miscellaneous

11. Gypsum in Agriculture

Older references 
Miscellaneous references

Bibliography of Olson and St. John

Bibliography of DeKalb

GYPSUM, GENERAL REFERENCES.

Annual Reports, Mineral Industry, U. S. Geol. Survey Reports.

Bericht uber die XVI Haupt Versammlung des Deutschen Gips vereins Berlin, 1914.

Cements, Limes and Plasters, Eckel, Edwin C., John Wiley \& Sons, Publishers, 1905. 2d. edition, 1922.

Der Gips, H. V. Waldegg, verlag von T.' Thomas, Leipsig, 1906.

Der Gips und Seine Verwendung, Pedrotti, 1901.

Der Stuck und Estrichgips, Physical-Chem. Untersuchungen, Rohland, 1904.

Die Gewinnung und Verwendung des Gipses, Moye, 1908.

Economic Geology of the United States, MacMillan, 1910, Chapter 7, Ries, $\mathrm{H}$.

Geology of Webster County, Iowa, Wilder, F. A., Iowa Geol. Survey, Vol. XII, 1902, p. 63.

Gips und Anhydrit, Van't Hoff, 1901.

Gypsum Deposits of the United States, U. S. Geol. Survey, Bull. 223, 1904, Adams, G. I., and others.

Gypsum Deposits of the United States, Stone, R. W., and others, U. S. Geol. Survey Bull., 697, 1920.

Gypsum, in Mineral Industry, Wilder, F. A., Vols. XXII, XXIII, XXIV', XXV, XXVI, XXVII, XXVIII, 1914, '15, '16, '17, '18, '19, '20, '21.

Gypsum Products, Stone, R. W., Bureau of Mines Technical Paper 155, 1917.

Gypsum and Salt in Oklahoma, Snider, L. C., Oklahoma Geol. Survey Bull. 11, p. 214, 1913.

Gypsum in Canada, Cole, L. H., Canada Dept. of Mines, 1913 , p. 145.

Gypsum and Plaster of Paris, Winterbottom, D C., South Australia Dept. of Chemistry, Bull. 7, p. 161, 1917.

Gypsum Industry of Michigan and the Plaster Industry, Grimsley, G. P., Geol. Surv. of Michigan, Vol. IX, Part 2, p. 243. 
Kalk, Zement und Gips, Die Bevertung und Anwendung, Tor$\min , 1905$.

Materials of Construction, Mills, A. P., John Wiley \& Sons, pp. 1-18, 1915.

Non-Metallic Minerals, Merrill, G. P., pp. 326-332, 1904.

Plastering, Plain and Decorative, Millar, Wm., Trumslove \& Comba, N. Y. Publishers, 1897.

Special Report of Gypsum and Gypsum Cement Plasters, Grimsley, G. P., University Geol. Surv. of Kansas, Vol. V. p. 183, 1899.

Studien uber Stuckgips, totgebrannten und Estrich gips, Glassenapp, 1908.

Untersuchungen uber gips, Muller, R., 1904.

\section{BIBLIOGRAPHIES}

GENERAL FOR UNITED STATES.

U. S. Geol. Survey Bull. 540, p. 380, 1914.

Cements, Limes and Plasters, Eckel, E. C., 1905.

Report on Gypsum in Canada; Canadian Dept. of Mines, Cole, L. H., 1913.

Dealing with Gypsum in Agriculture, Courtney DeKalb, Manufacturers' Record, Dec. 20, 1917.

Gypsum Industry in 1912, R. W. Stone, U. S. Geol. Survey, 1913.

State College of Washington. Bulletin 165; Olsen and St. John, 1921.

Gypsum Deposits of the United States, U. S. Geol. Survey Bull. 697, R. W. Stone, 1920.

Geological Survey of Michigan, G. P. Grimsley, Vol. IX, 1903-4.

LOCALITY REFERENCES

Adams, G. I.,

Burchard, E. F.,
Gypsum deposits in the United States: C. S. Geol. Survey Bull. 223, 1904.

The gypsum industry: U. S. Geol. Survey. Mineral Resources, 1909, pt. 2, 1910. 
The gypsum industry: U. S. Geol. Survey .Mineral Resources, 1910, pt. 2, 1911.

The gypsum industry: U. S. Geol. Survey Mineral Resources, 1911, pt. 2, 1912.

Loughlin, G. F., The gypsum industry in 1914: U. S. Geol. Survey Mineral Resources, 1914, pt. 2, 1915.

Stone, R. W., The gypsum industry: U. S. Geol. Survey Mineral Resources, 1912, pt. 2, 1913.

The gypsum industry: U. S. Geol. Survey Mineral Resources, 1913, pt. 2, 1914.

The gypsum industry: U. S. Geol. Survey Mineral Resources, 1915, pt. 2, 1916.

The gypsum industry: U. S. Geol. Survey Mineral Resources, 1916, pt. 2, 1917.

The gypsum industry: U. S. Geol. Survey Mineral Resources, 1917, pt. 2, 1918.

The gypsum industry: U. S. Geol. Survey Mineral Resources, 1918, pt. 2, 1919.

Stone, R. W., and others, Gypsum Deposits of the United States: U. S. Geol. Survey Bull. 697, 1920.

LOCALITY REFERENCES BY STATES.

ALASKA.

Burchard, E. F.,

Gypsum in Alaska: U. S. Geol. Survey Mineral Resources, 1913, p. $363, .1914$.

Wright, C. W., Nonmetallic mineral resources of southeastern Alaska: U. S. Geol. Survey Bull. 314, pp. 79-80, 1907. 
ARIZONA.

Blake, Wm. P.,

Culin, F. L., Jr.,

Lee, W. 'T.,

Stone, R. W.,

Hill, R. T.,

Miser, H. D.,

Gypsum Deposits in the United States: U. S. Geol. Surv. Bull. 223, 1904.

Gypsum: Univ. of Arizona Bull. 19, 8 pp., 1915.

Geologic reconnaissance of a part of western Arizona: U. S. Geol. Survey Bull. 352, p. 36, 1908.

Gypsum Deposits of the United States: U. S. Geol. Survey Bull. 697, pp. 49-56, 1920.

$A R K A N S A S$.

The Neozoic geology of southwestern Arkansas: Arkansas Geol.

. Survey, Vol. 2, pp. 119, 241, 257, 1888.

Gypsum Deposits of the United States: U. S. Geol. Surv. Bull. 697, p. 57, 1920 .

CALIFORNIA.

Aubury, L. E.,

Boyle, A. C.,

The structural and industrial minerals of California: Bull. Cal. State Min. Bur. No. 38, pp. 281-288; 1906. Geology and Ore Deposits of the Bully Hill Mining District, Am. Inst. Min. Eng. Bull. 90, pp. 1392-4. 1914.

Crawford, J. J., Gypsum (localities in California) California State Mineralogist, 12th Ann. Rept., 1894.

Fairbanks, H. W., . Gypsum deposits in California: U. S. Geol. Survey Bull. 223, 1904.

Grimsley, G. P., Gypsum and cement plant industry in California: Eng. \& Min. Jour., June 8, 1901. 
Hess, F. L.,

Hess, F. L.,

Surr, G.,

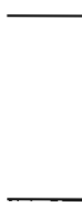

Burchard, E. F.,

Gilbert, G. K.,

George, R. D.,

Lee, H. A.,

Lakes, A.,

Lakes, A.,
BIBLIOGRAPHY

A reconnaissance of the gypsum deposits of California: U. S. Geol. Survey Bull. 413, 1910.

Gypsum deposits near Cane Spring, Kern County, California: U. S. Geol. Survey Bull. 430, pp. 417-18, 1910.

Gypsum deposits of the United States: U. S. Geol. Survey Bull. 697, pp. 58-86, 1920.

Gypsum deposits of the Maria Mountains: Mining World, April 15, 1911, pp. 787-790.

Brief description of gypsum in various counties: 12th and 13th Reports State Mineralogist, 1894 and 1896.

Gypsum deposits at Point Sal, Santa Barbara County: 8th Ann. Rept. State Mineralogist, pp. 538 and 688, 1888, and 10th Ann. Rept. p. 601, 1890 .

COLORADO.

Gypsum deposits of Eagle County: U. S. Geol. Survey Bull. 470, pp. 354-365, 1911.

U. S. Geol. Survey, Geol. Atlas, Pueblo folio, p. 6, 1897.

Gypsum deposits of the United States: U. S. Geol. Survey Bull. 697, pp. 87-94, 1920.

Larimer County gypsum: Stone, Vol. 21, pp. 36-37.

Gypsum deposits in Colorado: U.

S. Geol. Survey Bull. 223, 1904.

Gypsum and clay in Colorado: Mines and Minerals, Vol. 20, Dec. 1899. 
Ransome, F. L.,

Siebenthal, C. E.,

Spencer, A. C.,

Day, D. T.,

Stone, R. W.,

Stone, R. W.,

Wilder, F. 'A.,

Burchard, E. F.,

Stone, R. W.,

Kay, G. F.,
Ore deposits of the Rico Mountains: U. S. Geol. Survey, Twentysecond Ann. Rept. pt. 2, pp. 273280, 1901.

Gypsum deposits of the Uncompahgre region: U. S. Geol. Survey Bull. 285, pp. 401-3, 1906.

Geology of the Rico Mts.: U. S. Geol. Survey, Twenty-first Ann. Rept. pt. 2, pp. 48-54, 1900.

FLORIDA.

Gypsum deposits in Florida: U. S. Geol. Survey Bull. 223, 1904.

Gypsum in Florida: U. S. Geol. Survey, Twentieth Ann. Rept. pt. 6, 1899.

U. S. Geological Survey Bull. 697, pp. $95-98,1920$.

Gypsum in Florida: U. S. Geol. Survey Mineral Resources, 1918, pt. 2, pp. 293-296, 1919.

Florida Gypsum deposits explored : Rock Products, Vol. 23, No. 2, pp. 30-31, 1920.

Gypsum: Mineral Industry, 1917, p. 117, 1918.

IDAHO.

Gypsum industry in 1910: U. S. Geol. Survey Mineral Resources, 1910 , pt. 2, p. $727,1911$.

Gypsum deposits of the United States: U. S. Geol. Survey Bull. 697, pp. 99-100, 1920.

IOWA.

A New Gypsum Deposit in Iowa: U. S. Geol. Survey Bull. 580 pp. 59-64, 1915. 
Keyes, C. R.,

Lees, James H.,

Lees, James H., and Thomas, A. O.,

Stone, R. W., and Kay, G. F.,

Wilder, F. A.,

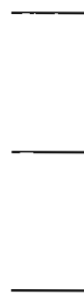

Bailey, E. H. S.,
Geological Age of Certain Gypsum Deposits: Am. Geologist, Vol. 30, pp. 99-102, 1902.

Gypsum Deposits in Iowa: Iowa Geol. Survey, Vol. III, 1895.

Iowa Gypsum: Mineral Industry, Vol. 4, pp. 379-396, 1896.

Miocene Age of Fort Dodge Gypsum: Eng. and Min. Jour., Vol. 100, p. 466, Sept. 18, 1915.

Some Features of the Fort Dodge Gypsum: Iowa Acad. Science, XXV, pp. 587-598, 1918.

The Structure of the Fort Dodge Beds: Iowa Acad. Science, XXIX, 1922.

The Ste. Genevieve Marls near Fort Dodge and their Fauna: Iowa Acad. Science XXV, pp. 599-616, 1918.

Gypsum Deposits of the United States: U. S. Geol. Survey Bull. 697, pp. 101-110, 1920.

Geology of Webster County: Iowa Geol. Survey, Vol. XII, pp. 63-235, 1902.

The Age and Origin of the Gypsum of Central Iowa: Jour. Geol., Vol. 11, pp. 723-748, 1903 .

Gypsum Deposits in Iowa: U. S. Geol. Survey Bull. 223, pp. 49-52, 1904.

Fort Dodge as a Plaster Center: Rock Products, July 22, 1910.

KANSAS.

Special Repòrt on Gypsum and Gypsum Cement Plasters: Kansas Univ. Geol. Survey, Vol. V, 1899. 
Bailey and Whittier,

Crane, W. R.,

Grimsley and Bailey,

Grimsley, G. P.,

Haworth, Erasmus,

Prosser, C. S.,
On the Chemical Composition of some Kansas Gypsum Rocks: Kansas Univ. Quarterly, Vol. 6, 1897. Mining and Milling of Gypsum in Kansas: Eng. and Mining Jour., Nov. 9, 1901.

The Gypsum Plaster Industry of Kansas: Eng. and Min. Jour., Vol. 77, pp. 442-448, 1904.

Gypsum in Kansas: Kansas Univ. Geol. Survey, Vol. V, 183 pp., 1899. Gypsum in Kansas: Kansas Univ. Quart., Vol. 6, pp. 15-27, 1897.

Gypsum Deposits of Kansas: Bull. Geol. Soc. America Vol. 8, pp. 227-240, 1897.

Cost of Plaster Manufactured at Kansas Mills: Mineral Industry, Vol. 7, p. 392.

Gypsum Deposits of Kansas: Mineral Industry, Vol. 6, pp. 395-6, 1897.

Gypsum Deposits in Kansas: UT. S. Geol. Survey Bull. 223, 1904.

Minéral Resources of Kansas: Kansas Univ. Geol. Survey, Ann. Bulls., 1898, pp. 59-65, 1899, pp. $54-55$.

Gypsum Deposits of the U. S.: U. S. Geol. Survey Bull. 697, pp. 111-120, 1920.

The Upper Permian: Kansas Univ.

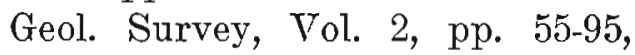
1897.

\section{LOUISIANA.}

Harris, G. D.,

Stone, R. W.,
Oil and Gas in Louisiana: U. S. Geol. Survey Bull. 429, 1910. Gypsum deposits in the United 
States: U. S. Geol. Survey Bull. 697, p. 121, 1920.

Gregory, W. M.,

Grimsley, G. P.,

Smith, R. A.,

Rowe, J. P.,

Freemen, O. W.,
MICHIGAN.

Gypsum in Arenac and adjoining counties: Ann. Rept. Michigan Geol. Survey, 1901, pp. 15-18.

The Alabaster Area: Michigan Geol. Survey, Vol. 9, pt. 2, pp. 6077, 1904.

A Theory of Origin for the Michigan Gypsum Deposits; American Geologist, Vol. 34, pp. 378-387, Dec., 1904.

The Gypsum Industry of Michigan and the Plaster Industry: Geol. Survey of Michigan, Vol. IX, pt. 2, p. 243.

A Preliminary Report on the Gypsum Deposits of Michigan: Michigan Geol. Survey for 1902, pp. 4-10. Gypsum Deposits in Michigan: UT. S. Geol. Survey Bulletin 223, 1904. Gypsum Deposits of the United Stàtes: U. S. Geol. Survey Bull. 697, pp. 122-129, 1920.

MONTANA.

Gypsum and Lime Industry in Central Montana: Min. and Eng. World, Vol. 45, pp. 663--664, 1916. Montana Gypsum Deposits: Am. Geologist, Vol. 35, pp. 104-113.

Gypsum Deposits of Montana: Eng. and Min. Jour., Vol. 85, p. 1243, 1908.

Montana Gypsum Deposits: Mines and Minerals, Vol. 28, pp. 59-60) 1907. 
Stone, R. W.,

Weed, W. H.,

Higgins, W. C.,

Jones, J. C.,

Louderback, G. D.,

Rogers, A. F.,

Darton, N. H.,

Herrick, C. L.,

Brady, F. W.,
Gypsum Deposits of the United States: U. S. Geol. Surv. J3ull. 697, pp. 131-138, 1920.

Gypsum Deposits in Montana: UT. S. Geol. Survey Bull. 223, 1904.

NEVADA.

The Nevada Douglas Copper Mining Co.' Gypsum Deposits: Salt Lake Min. Review, p. 15, Mar. 30, 1913.

Origin of the Anhydrite at the Ludwig Mines: Econ. Geology, Vol. 7, pp. 400-402, 1912.

Gypsum Deposits of the United States: U. S. Geol. Survey Bull. 697, pp. 139-160, 1920.

Gypsum Deposits in Nevada: U. S. Geol. Survey Bull. 223, pp. 112118, 1904.

Occurrence and Origin of Gypsum and Anhydrite at the Ludwig Mine: Econ. Geology, Vol. 7, pp. 185-189, 1912.

NEW MEXICO.

The White Sands of New Mexico: Mines and Minerals, Vol. 25, pp. 529-530, 1905.

Gypsum Deposits of the United States: U. S. Geol Survey Bull. 697, pp. 161-186, 1920.

Gypsum Deposits in New Mexico: U. S. Geol. Survey Bull. 223, 1904. Geology of the White Sands of New Mexico: Bull. Hadley Jaab. Univ. New Mexico, Vol. '2, pt. 1. Geology of the White Sands: New Mexico: Jour. Geology Vol. 8, 1900, pp. 112-128. 
Newell, F. H.,

Shaler, M. K.,

Beck, L. C.,

Clark, W. C.,

Eckel, E. C.,

Hall, James,

Leighton, Henry,

Lincoln, D. F.,

Luther, D. D.,

Merrill, F. J. H.

Newland, D. H.,

Newland and Leighton,

\section{BIBLIOGRAPHY}

The Gypsum Plains: U. S. Geol. Survey Twelfth Ann. Rept., pt. 2, pp. 281-282, 1891.

Gypsum in northwestern New Mexico: U. S. Geol. Survey Bull. 315, pp. 260-266, 1907.

Gypsum in Northwestern New Mexico: Eng. and Min. Jour., Yol. 83, p. $1091,1907$.

NEW YORK.

Mineralogy of New York: pp. 6167, 237-238, 1842.

Gypsum Industry of New York State: New York State Mus. Bull. 11, 1893, pp. 70-84.

Gypsum Deposits in New York: U. S. Geol. Survey Bull. 223, 1904.

Geology of New York, pt. 4, pp. 421-457, 1843.

Gypsum Deposits of New York State: New York State Mus. Bull. 143, 94 pp., 1910.

Report on Seneca County, N. Y.: New York State Geologist, Fourteenth Ann. Report, 1896.

The Economic Geology of Onondaga County: Rept. New York State Geologist, Vol. I, 1897.

Salt and Gypsum Industries in New York State: N. Y. State Museum Bull. 11, 18,93.

Gypsum Deposits of New York State: New York State Mus. Bull. 15, 1895.

The Mining and Quarry Industry of New York State: New York State Mus. Bulletins 1905 to 1917. Gypsum Deposits in New York 
Parsons, A. L.,

Pohlman, J.,

Williams, S. G.,

Gibson, H. G.,

Orton, E.,

Peppel, S. V.,

Gould, C. N.,
State: New York State Mus. Bull. 143, 1910.

Gypsum Deposits of the United States: U. S. Geol. Survey Bull. 697, pp. 187-217, 1920.

Recent Developments in the Gypsum Industry of New York State: New York State Geologist, 20th Ann. Rept., 1902, pp. 177-183.

Notes on the Gypsum Deposits of New York: 57th Ann Rept. New York State Museum, Vol. I, pp. 89-157, 1905.

Cement Rock and Gypsum Deposits in Buffalo, New York: Trans. Am. Inst. Min. Eng., Vol. 17, pp. 250253, 1889.

Age of Gypsum Deposits of New York: Am. Jour. Science, Sept., 1885.

Gypsum Deposits of Cayuga County: Am. Jour. Science, Vol. 130 , p. 212.

OHIO.

Bownocker, J. A.,

Gypsum Deposits of the United States: U. S. Geol. Survey Bull. 697, pp. 218-223.

Unwatering a Gypsum Mine at Centralia: Min. and Eng. World, Vol. 41, p. 955, Nov. 21, 1914. Gypsum or Land Plaster in Ohio: U. S. Geol. Survey, Vol. 8, 1888.

Gypsum Deposits in Ohio: U. S. Geol. Surv. Bull. 223, 1904.

OKLAHOMA.

Gypsum Deposits in Oklahoma: U. S. Geol. Survey Bull. 223, 1904. 
Oklahoma Gypsum: Oklahoma Dept. Geology, Second Bienn. Rept. pp. 75-137, 1902.

Geology and Water Resources of Oklahoma: U. S. Geol. Survey Water Supply Paper 148, 1.905. Preliminary report on the Structural Materials of Oklahoma: Oklahoma Geol. Survey Bull. 5, pp. 98-113, 1911.

Extent and Importance of Oklahoma Gypsuma Deposits: Min. Sci. Vol. 56, pp. 542-543, 1907; pp. 583584, 1907.

Snider, L. C., Gypsum and Salt in Oklahoma: Olslahoma Geol. Survey Bull. 11, 214 pp. 1913.

Gypsum Deposits of the U. S.: U. S. Geol. Survey Bull. 697, pp. 224235, 1920.

Oklahoma Gypsum Deposits and Industry: Eng. and Min. Jour. Vol. 95, pp. 931-933, 1913.

OREGON.

Lindgren, W.,

Gypsum Deposits in Oregon: U. S. Geol. Survey Bull. 223.

Stone, R. W., Gypsum Deposits of the U. S.: U. S. Geol. Survey Bull. 697, pp. 236238, 1920 .

SOUTH DAKOTA.

Darton, N. H.,

Preliminary Description of Geology of Southern half of Black Hills, etc.: U. S. Geol. Survey 21st Ann. Rept. pt. 4, pp. 517-518, 584-585, 1901.

Gypsum Deposits of South Dakota: U. S. Geol. Survey Bull. 223, 1904. 
Preliminary Report on Geology and Underground Water Resources of Central Great Plains: U. S. Geol. Surrey Prof. Paper 32, pp. 392-394, 1905.

Hutton, J. G.,

Richardson, G. Bi.,

Cummins, W. F.,

Gould, C. N.,

Hill, B. F.,

Richardson, G. B.,

Stone, R. W.,

Udden, J. A.,
Gypsum Deposits of the U. S.: U. S. Geol. Survey Bull. 697, pp. 239249, 1920.

Upper Red Beds of the Black Hills: Jour. Geology, Vol. 11, pp. 365-393, 1903.

$T E X A S$.

Oil and Gas Fields of the Western Interior and Northern Texas Coal Meàsures, etc.: U. S. Geol. Survey Bull. 184, pp. 49-53, 1901.

Geology of Nortlwestern Texas: Texas Geol. Survey 2nd Ann. Report, pp. 455-459, 1891.

Geology and Water Resources of Oklahoma: U. S. Geol. Survey Water Supply Paper 154, 1906.

Geology and Water Resources of Western Panhandle of Texas: U. S. Geol. Survey, Water Supply Paper 191, 1907.

Gypsum Deposits of Texas: U. S. Geol. Survey Bull. 223, pp. 68-73.

Salt, Gypsum and Petroleum in trans-Pecos Texas: U. S. Geol. Survey Bull. 260, pp. 573-585, 1905.

Gypsum Deposits of the United States: U. S. Geol. Survey Bull. 697, pp. 250-260, 1920.

The Deep Boring at Spur: Texas TTniv. Bull. 363, 1914. 
OTAH.

Boutwell, J. M.,

Gilbert, G. K.,

Lupton, C. T.,

Stone, R. W., and

Lupton, C. T.,

Talmage, J. E.,

Boyd, C. R.,

Eckel, E. C.,

Robertson, W.,

Stone, W. H.,

Stone, Wm.'H.,

Stose, G. W.,
Rock Gypsum at Nephi, Utah: U. S. Geol. Survey Bull. 225, pp. 483487, 1904.

Gypsum Deposits of Utah: U. S. Geol. Survey Bull. 223, 1904.

Lake Bonneville: U. S. Geol. Survey Mon. I, pp. 222-223, 1890.

Gypsum along the west flank of the San Rafael Swell: U. S. Geol. Survey Bull. 530, pp. 221-231, 1913. Gypsum Deposits of the United States: U. S. Geol. Survey Bull. 697, pp. 261-282, 1920.

A remarkable occurrence of selenite: Science, Vol. 21, pp. 85-87. 1893.

VIRGINIA.

Mineral Resources of Southwest Virginia, 1881.

Gypsum Deposits in Virginia: U. S. Geol. Survey Bull. 223, 1904.

Salt and Gypsum Deposits of Southwest Virginia: U. S. Geol. Survey Bull. 213, p.p. 406-417, 1903. Some notes on the Holston Salt and Gypsum: The Virginias, Vol, 3, pp. 20-42, 1882.

Gypsum Plant in Southwestern Virginia: Mfrs. Record, Aug. 20, 1908, pp. 51-52.

New Plant of Southern Gypsum Co., Cement and Eng. News, Vol. 20, pp. 293-294, 1908.

Gypsum Deposits of the United States: U. S. Geol. Surv. Bull. 697, pp. 283-294, 1920.

Geology of the Salt and Grvpum 
Deposits of Southwestern Virginia: U. S. Geol. Survey Bull. 530, pp. 232-255, 1913.

Sterenson, J. J.

Watson, T. L.,

Branson, E. B.,

Darton, N. H.,
The Salt and Gypsum Deposits of the Holston Valley: The Virginias, Vol. 6, pp. 53-55, 1885.

Notes on the Geological structure of Tazewell, Russell, Wise, Smyth, and Washington Counties: Amer. Phil. Soc. Proceedings, Vol. 22, 1885.

Mineral Resources of Virginia: Virginia Jamestown Exposition, pp. 327-335, 1907.

\section{WYOMING.}

Origin of Red Beds of western Wyoming: Geol. Soc. America Bull., Vol. 26, pp. 217-230, 1915.

Preliminary description of the geology and water resources of the southern half of the Black Hills and adjoining regions in South Dakota and Wyoming: U. S. Geol. Survey Twenty-First Ann. Rept., pt. 4, pp. 516-518, 584585, 1901.

U. S. Geol. Survey Atlas, Newcastle folio (No. 107), p. 9, 1904. Preliminary report on the geology and underground water resources of the central Great Plains: U. S. Geol. Survey Prof. Paper 32, pp. 392-394, 1905.

and O'Hara, C. C., U. S. Geol. Survey Geol. Atlas, Aladdin folio (No. 128), p. 8, 1905. Geology of the Owl Creek Mountains: 59th Cong. 1st. sess., S. Doc. 219, p. 19, 1906. Paleozoic and Mesozoic of central 
Wyoming: Geol. Soc. America Bull., Vol. 19, pp. 403-470, 1908.

- and Siebenthal, C. E., Geology and mineral resources of the Laramie Basin, Wyoming: U. S. Geol. Survey Bull. 364, 1909.

Fisher, C. A.,

Jamison, C. E., Mineral Resources of the Big Horn Basin: U. S. Geol. Survey Bull. 285, p. 313, 1905.

Geology and water resources of the Big Horn. Basin, Wyoming: U. S. Geol. Survey Prof. Paper 53, p. 18, 1906.

Geology and mineral resources of a portion of Fremont County, Wyoming: Wyoming Univ. Bull. 2, ser. B, p. 84, 1911.

Knight, W. C., Gypsum Deposits in Wyoming: U. S. Geol. Survey Bull. 223, pp. 7985, 1904.

Gypsum deposits of Taramie Plains: Franklin Institute Jour., Vol. 157, p. 745, 1904.

Lupton, C. T., and Condit, D. D.,

Gypsum in the southern part of the Big Horn Mountains, Wyoming: U. S. Geol. Survey Bull. 640, pp. 139-1.57, 1916.

Mouldy, R. B., and Slosson, E. E., The - Laramie cement plaster: Wyoming Agr. Coll. Tenth Ann. Rept., 1900.

Richardson, G. B., Tpper Red Beds of the Black Hills: Jour. Geology, Vol. 11, pp. 365393, 1903.

Siebenthal, C. E., Gypsum deposits of the Laramie district, Wyoming: U: S. Geol. Survey Bull. 285, pp. 404-405, 1906.

Stone, R. W., Gypsum deposits of the United States: U. S. Geol. Survey Bull. 697, pp. 295-308, 1920.

Slosson, E. E., .. The Laramie Cement Plaster: 10th 
Trumbull, L. W.,

Bailey and Ells,

Cole, L. H., and Jennison, W. F.,

Fletcher, H.,

Gesner, A.,

Jennison, W. F.,

Kramer, H. F.,

Tyrrell, J. B.,

Tyssowski, J.,
Ann. Rept., Wyoming Agr. College, 1900 .

Cement Plaster industry in Wyoming: Min. World, Vol. 26, p. 387, 1907.

FOREIGN.

CANADA.

Report on Lower Carboniferous of Albert and Westmoreland Counties, New Brunswick: Canadian Geol. Survey Report for 1876-77-78.

Gypsum in Canada, Dept. of Mines, 256 pp., 1913.

Gypsum Industry of Ontario and Western Canada: Summary Rep. Mines Branch, Dept. of Mines Canada, for 1911.

Gypsum in various Provinces: Index to Geol. Survey of Canada Repts. 1863-1884 and 1885-1906.

Explorations and Surveys in Cape Breton, Nova Scotia: Canada Geol. Survey Rept. for 1875-6, pp. 369-418.

On the Gypsum of Nova Scotia: Quarterly Journal Geol. Soc., Vol. 5, pp. 129-130, 1849.

Gypsum Deposits of the Maritime Provinces: Pub. No. 84, Mines Branch, Dept. of Mines, Canada. Gypsum of New Brunswick: Canada Geol. Survey Rept. 1911, pp. 322-28.

Gypsum Deposits in Northern Manitoba: Canadian Record of Seience: Vol. III, 1888-9.

Gypsum on Cape Breton Island, 
Wilhoit,

Rangel, M.,

Kemp, W. J., and Lewis, G. A., Metcalf, H. T.,

LaCroix, A.,

Boyer, J.,

Gary,
Nova Scotia: Eng. and Min. Jour., Vol. 88, p. 569.

The Mineral Wealth of Canada, Dawson's Acadian Geology, pp. 105-111, 1897.

Origin of Gypsum of Plaster Cove, Nova Scotia: Quarterly Journal, Geol. Soc., Vol. V, p. 339.

Gypsiferous Formation, Nova Scotia: Quarterly Journal, Geol. Soc., Vol. VIII, pp. 398, 399.

Gypsum in Cape Breton, Nova Scotia: Quarterly Journal, Geol. Soc., Vol. I, pp. 211-212; Vol. V, pp. 335-339; Vol. XLII, p. 523.

Gypsum, Nova Scotia: Canadian Mining Review, March, 1896.

MEXICO.

Gypsum in Durango: Eng. and Min. Journal, July 30, 1921.

ENGLAND.

Gypsum in Sussex: Inst. of Min. Eng., Trans., Vol. 33, p. 449.

Gypsum deposits in Nottingham-. shire and Derbyshire: Inst. Min. Eng., Trans., Vol. 12, p. 107. FRANCE.

Les formes du Gypse des environs de Paris: Bull. de la Soc. Francais de Min., 1898, 31, 39.

Gips, Pariser (Vorkommen, Analysen, Verwendung): Thonind, Vol. 25, pp. 1678-1680, 1901.

Plaster mining in the vicinity of Paris: Eng. Mag., pp. 850-859, 1906. Gips, Pariser: Mitteilungen Versuch, Vol. 19, p. 7, 1901. 
GERMANY.

Wilder, F. A.,

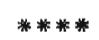

***

Winterbottom, D. C.,

Opstaele, Geo.,

Hume, W. F.,
Bericht uber die XVI Haupt Versammlung des Deutschen Gips Verein, Berlin, 1914.

Gypsum Industry in Germany: Geol. of Webster Co., Iowa Geological Survey, Vol. XII, pp. 195222, 1902.

Gips in Sperenberg und seine technische verwendung. Tonind. Zeitung, p. 111, Jan. 20, 1912.

Aus der sud deutschen Gipsindustrie. Tonind. Zeitung, July 30, 1912.

SWITZERLAND.

Dir Gipsindustrie du Schwiz. Tonindustrie Zeitung, Apr. 6, 1912.

AUSTRALIA.

Gypsum and Plaster of Paris: South Australia Dept. of Chemistry Bull. 7, 661 pp. 1917.

Australien, Gips in: Tonindustrie Zeitung, Vol. 31, pp. 186-187, 1907.

EGYPT.

Exploitation platriere moderne en Egypte: Rev. d. Materiaux, Dec. 1911, p. 205.

Gypsum Deposits : of Mariut Region (Mediteranean Egypt) : Cairo Scient. Jour., Feb., 1912, p. 43.

\section{GEOLOGY}

Die Bildung der Steinsalzlager und Mutter langen. C. Ochsenius, Halle, 1877.

Genetische Beziehungen Zwischen Gyps und Anhydrit: Kissivan, Thonind., Vol. 20, p. 906. 
Gipslagen, Wo finden sich und wie enstehen, Fiebelkorn: Thonind., Vol. 25, pp. 326-330, 1901.

Origin of Gypsum: 'T. Sterry H.unt, Chem. and Geol. Essays, Chapt. V.III.

Origin of the Red Beds of Western Wyoming: E. B. Branson, Bull. Geol. Soc. America, Vol. 26, pp. 217-242.

Some Conclusions in regard to the Origin of Gypsum: F. A. Wilder, Bull. Geol. Soc. America, Vol. 32, pp. 385-394, 1921. Theories of Origin of Gypsum: Sherwin, Trans. Kansas Acad. Science, Vol. XVIII, pp. 85-88, 1903.

Uber dies Beschaffenheit und entstehung. Parallel faseriger aggregate von Stein salz und von Gips: R. Schmidt, Kali, April, 1914, p. 161.

\section{CHEMICAL \& MINERALOGICAL}

Ammoniumsyngenit, D'Ans-(Loslichkeit von Gips in Ammoniumsulfatlosungen): Berichte Chemischen Gesellschaft, Vol. 39, pp. 3326-3328, 1906.

Albatre, Influence de la Temperature de deshydrateation de l', sur la prise du platre obtenu: Ieduc et Pellet, Comptes Rendu, Vol. 143, pp. 317-320, 1906.

Alten Gips durch Schwefel Zu regenieren: Belargent, Brev. d'invention, T. 17, p. 283, Bulletin des sciences technical, T. 18, p. 89, Dingless pol Jourie, Bd. 44, S. 154.

Calcium Sulphate in Aqueous solutions: Cameron and Bell, U. S. Department of Agriculture, 1906.

Calcium sulphate and alteration characteristics of gypsum and anhydrite: Zeit fur Anorg. Chemie, Vol. 14, p. 1072.

Chemical Composition of Some Kansas Gypsum Rocks: Bailey and Whittier, Kansas Univ. Quarterly, Vol. 6, 1897. Calciumsulfat and Tmwandungsbedingungen von Gips und Anhyrite: Weigert, Physikalische Zeitschrift, Vol. 3, p. 190, 1902.

Chemical Tests of Gypsum: Alfr. Cavazzi, Ann. Chem. Applicata, Vol. 6, pp. 18-26, 1916; reviewed in Chem. Abstr. Vol. 10, No. 22, p. 2975, 1916.

Calciumsulfats, die Zweite anhydritische Modifikation des: Rohland, Zeit. Anorganische Chemie, Vol. 35, pp. 194-204. 
Calciumsulfats, die erste anhydritische Modifikation des: Rohland, Zeit. Anorganische Chemie, Vol. 36, pp. 332-339, 1903.

Calciumsulfats, Hydration des Hemihydrats des: Kosmann, Tonindustrie Zeitung, Vol. 27, pp. 1735-1737, 1903.

Die /Hydration des Gipses: Rohland, Tonindustrie Zeitung, Vol. 28, pp. 342-344, 389, 392, 1904.

Der Stuck und Estrichgips physikalisch-chemische Untersuchungen: P. Rohland, Leipsic, 1904, Verlag Quandt und Handel.

Die Steinsalz industrie von Stassfurt und umgegend: H. Piecht, 5 Aufl, 1891, Stassfurt, Verlag Weicke.

Disidratazione della selenite e idiatazione dell auldrite. Gaz. Chim. Ital. 30, pp. 333-339, Zunino.

Determination of Water and Sulphuric Acid in Gypsum: Storer, Chemical News, Vol. XXII, p. 99.

Die Wellenlange des Reststrahlen von: Koch, Annalen der Physik, Vol. 26, pp. 974-984, 1908.

Estrichgips (Die Hydration des Anhydrids): Rohland, Tonindustrie Zeitung, Vol. 27, pp. 1145-1148, 1903.

Formation d'anhydrite per Calcination der Gypse a haute temperature: Lacroix, Compte Rendue Vol. 126, pp. 533534.

Gips, Unloslichmachen von, in Wasser (Durch Hinzufugen von borsaurem Ammon) : Am. Apoth. Z., Vol. 23, p. 131, 1902. Gipsmortels, das Treiben des: Rohland, Tonindustrie Zeitung, Vol. 28, pp. 1297-1300, 1904.

Gips und Anhydrit: Van't Hoff, Armstrong, Henrichsen, Weigert und Just, Z. Physik. Chem., Vol. 45, pp. 257-306, 1903.

Gips (Hydratwasserhaltige Verbindungen und anhydridische Modifikationen) : Moye, Chemiker Zeitung, Vol. 30, pp. 544$545,1906$.

Gipses, Weitere Bestahigung der Beziehung Zwischen der. Aenderung der Loslichkeit des, und seiner Abbindezeit: Rohland, Tonind. Zeit., Vol. 30, pp. 492-493, 1906.

Gipses und Portlandzements, Hydratation des, Beziehungen Zwischen der Loslichkeit des Calciumsulfates und: Rohland, Z. Ang. Chem., Vol. 18, pp. 327-330, 1905.

Gypsum, Solubility of, as Affected by Size of Particles and by 
Different Crystallographic Surfaces: Hulett, Amer. Chem. Soc. Jour., Vol. 27, pp. 49-56, 1905.

Gips und Anhydrit: J. H. Van't Hoff, E. J. Armstrong and others. Zeitschrift f. Physikal Chemie, 1903, XLV Band 3 Heft, Leipsic, Verlog W. Engelman.

Gips und Anhydrit, 1901. Van't Hoff.

Gipses, Loslosen erharten (Mit Einer schwachen Salzsaurelosung): Haarmann's Z. Vol. 47, pp. 167-168, 1903.

Gips, Uber, Van't Hoff: Zeit. fur Elektrochem., Vol. 8, pp. 575-579; Thonind., Vol. 26, pp. 429-431, 1902.

Gypsum, Solubility of: Hulett and Allen. Amer. Chem. Soc. Jour., Vol. 24, pp. 567-579, 1902.

Gipslosungen, gesattigte als Basis fur Leitfahigkeit: Hulett, Z. Physik. Chem., Vol. 42, pp. 577-583, 1903.

Gypse, Solubilitie du dans les Dissolutions de Sel Marin. Cuisson du Gypse. Prise du Platre: Soc. Chim. Bull., Vol. 29, pp. 167-174, 1903.

Gips, Totgebrannter: Moye, Tonindustrie Zeitung, Vol. 27, pp. 1820-1821, 1903.

Hydrat Wasser und gipsanalyse: Moye, Tonindustrie Zeitung, Apr., 13, 1912, p. 630.

Lime, Gypsum, Water, at $25^{\circ}$, System: Cameron and Bell, Am. Chem. Soc. Jour., Vol. 28, pp. 1220-1222, 1906.

Losungserscheinungen am anhydrit: W. Burkhardt. Zeits. fur Krystallogr. u. Mineralogie, Vol. 50, No. 3. Reviewed in Kali, May 15, 1912, p. 237.

Note on the Hydration of Anhydrite and dead burned gypsum: A. C. Gill, Jour. A. Cer. Soc., Jan., 1918.

Occurrences of Anhydrite in the U. S.: A. F. Rogers, Columbia School of Mines Quarterly, Vol. 36, pp. 139-142, Jan., 1915.

Optical Constants of, at Different Temperatures, and the Mitschenlich Experiment: Tutton, Proc. Royal Society, Vol. 81, Ser. A., pp. 40-57, 1908.

Plaster of Paris, Nature of Changes involved in Production and Setting of: Davis, Chem. Ind., Vol. 26, pp. 727-728, 1907. P̈latre en poudre, four destine a la cuisson du, Riviere et Vieu: Mon. Cer., Vol. 33, p. 98, 1902.

Plaster of Paris: L. A. Keane, Jour. Phys. Chem., Vol. 20, pp. 701-723, Nov., 1916. 
Recherches Experimentales sur la constitution des mortiers hydrauliques, M. H. Le Chatelier. 2 Edition 1904, Paris. Published by Viech Dunod.

Recherches sur le Platre: DeForcrand, Societe Chimique de Paris., Vol. 35, pp. 781-790, 1906.

Solubility of Gypsum: Hulett and Allen, Amer. Chem. Soc., Vol. 24, pp. 667-679, 1902.

Stuckgips (Hydratation des Hemihydrates): Rohland, Tonindustrie Zeitung, Vol. 27, pp. 675-680, 1903.

Some difficulties in the analysis of gypsum: G. Surr, Min. and Eng. World, Feb. 24, 1912, p. 467.

Solubility in sea water: A. Marmelli, Am. Chim. Applieata, Vol. 5, pp. 13-24, 1916; also J. Chem. Soc. London, Vol. 110, No. 11, p. 251, 1916.

Sulfate de Calcium Hydrate, Solubilite du, dans les Solutions de sel marin: D'Anselme; Soc. Chim. Bull. Vol. 29, pp. 372374, 1903.

The Reduction of Calcium Sulphate by Monoxide and Carbon: H. O. Hoffman, Trans. Am. Inst. Min. Engrs., Vol. XLI, 763-785.

The Nature of the Changes Involved in the Production and Setting of Plaster of Paris: Jour. Soc. Chem. Ind., Vol. 26, pt. 2, 1907.

The Behavior of Calcium Sulphate at Elevated Temperatures with some fluxes: H. O. Hoffman, Trans. Am. Inst. Min. Engrs., Vol. XXXIX, pp. 628-653.

Unos cristales gigantes de Yeso, Procedentes de la Mina naica Chihuahua: E. Wittich, Boletin Soc. Geol. Mex., Vol. 8, pt. 1, p. 61, 1913.

Untersuchungen uber Gips., Rich. Müller, Berlin, 1904, Verlog Tonindustrie-Ztg.

Uber die hydraulischen Eigenschaften des in der Gluhhitze behandleten Gipses: J. Schott, Polytechn. Jour., 1871, p. 355.

Uber in Hydration des Gipses, 1901, Rohland.

\section{TECHNOLOGY}

Backofen mit Dampfheizung Zum Brennen von Gips, aus welchem Grunde haben die, bisher keine Verwendung gefunden: Cramer, Thonind., Vol. 25, pp. 484-488, 1901. 
Burning Gypsum in Rotary Tube Furnaces, German Patent No. 287301, Jan. 8, 1914, F. M. Meyer.

Calcining of Gypsum: Eng. and Min. Jour., Vol. 96, p. 1220, 1913.

Einfluss der Hohe und dauer der temperatur beim gipskochen: Tonindustrie Zeitung, Vol. 98, p. 1177-81.

Gipsbrennofen: Gary, Thonind., Vol. 22, p. 707.

Gipskochapparat, der Opitz und Kaisersche: Opitz, Tonindustrie Zeitung, Vol. 29, pp. 821-827, 1905.

Gipses, Die Versuche Le Chateliers uber das Birennen, Abbinden und Erharten des: Tonindustrie Zeitung, Vol. 29, pp. 469-473, 1905.

Gips, Uber (Temperatur beim Erharten, einvirkung Kochsalz, Temperatur Wassers): Port Corresp. Zahn., Vol. 35, ; pp. 18-23, 1905.

Gipssteinbruche, Sprengmittel fur: Schimpf, Stein und Mortel, Vol. 11, p. 126, 1907.

Gips, Brennen von: Moye, Tonindustrie Zeitung, Vol. 31, pp. 782-783, 1907.

Gipskochapparate, Erfahrungen mit dem Petry-Heckingschen: Thonind., Vol. 26, pp. 476-478, 1902.

Gips (Brenntemperaturen), hydraulische Modifikationen: Rohland, Chemicker Zeitung, Vol. 26, pp. 804-806, 1902.

Gipskochapparat, der Petry-Hecking'sche, Wie hat sich, in der Praxis bewahrt: Koster, Thonind., Vol. 25, 'pp. 1678-1680, 1901.

Gips und Gipsindustrie: Zechner, Prometheus, Vol. 12, pp. 161-166, 1901.

Gypsum Plaster from the Ground to the Wall: E. C. Damron, Jr., Jour. Soc. Constructors Public Buildings, Vol. 4, pp. 272-290, 1913.

Gypsum Deposits, Manufacture and Mining: Eng. and Min. Jour., Vol. 72, p. 602; Vol. 82, p. 1063.

Gypsum Mining: W. J. Jones, Mines and Minerals, June, 1909, pp. 29 and 490.

Gypsum Mining and Milling in Kansas: W. R. Crane, Eng. and Min. Jour., Nov. 9, 1901.

Plaster of Paris or Stucco, Manufacture of, from Gypsum 
Rock: C. O. Bartlett, Cement and Eng. News, Vol. 20, pp. 121-123, 1908.

Plaster, Slaking of, and Means of Retarding its Hardening: Scientific American Suppl., Vol. 63, p. 26207, 1907.

Plaster of Paris, Manufacture of: Bartlett, Eng. \& Min. Jour., Vol. 82, pp. 1063-1064; Eng. Record, Vol. 53, p. 43 (suppl.) 1906.

The Stripping, Quarrying and Mining of Gypsum: L. H. Cole, Canadian Eng., Vol. 27, pp. 780-783, Dec. 24, 1914.

Technology of Cement Plasters: P.' Wilkinson, Trans. Amer. Inst. Mining Engrs., Vol. 27, pp. 508-519.

Unwatering a Gypsum Mine at Centralia, Ohio: H. G. Gibson, Eng. and Min. World, Vol. 41, pp. 955-956, 1914.

\section{STRUCTURAL}

Bericht uber die Prufung von Gips: Thonind., Vol. 25, pp. 778-9.

Cast in Place Reinforced Gypsum Roof Slabs on Paper Mill Building, Engineering News Record, November 1, 1917, Vol. 79, No. 18.

Calcareous Cements, Their Nature and Uses, Redgrave, 1895.

Der Gips als Baumaterial: Rombugs Leitscher J. Prak. Bank. pp. 19-32, 1862.

Essais sur la platre: E. Leduc and Maurice Pellet, Bulletin du Laboratoire d'Essais, Nov. 11., 1907.

Fireproofing with Gypsum: Virgil G. Marani, Construction, February, 1916.

Gypsum as a Fireproofing Material: Virgil G. Marani, Journal of Cleveland Engineering Society, Vol. 7, No. 3, November, 1914.

Gypsum Extended to Load Bearing Building Units: Virgil G. Marani, Rock Products and Building Materials, March 22, 1917.

Gypsum and its Effect on Imbedded Steel: Virgil G. Marani, Engineering News, August 3, 1916.

Gypsum, Its Part in the Repair Program: Virgil G. Marani. Building Supply News, November, 1918.

Gypsum in Present Day Modern Construction: Virgil G. Marani, Engineering and Cement World, January I, 1918. 
Gypsum as an Industrial Fire Resistant Material: Virgil G. Marani, Fire and Water Engineering, November 24, 1920. Gypsum: 'Products as Building Factors: Virgil G. Marani, Chicago Journal of Commerce, March 25, 1921.

Gypsum Floor and Roofs: Virgil G. Marani, Journal of American Institute of Architects, May, 1921.

Gypsum and its Products: Virgil G. Marani, Permanent Builder, September, 1920.

Gypsum Plaster Affords Fire Protection. Virgil G. Marani, Permanent Builder, 1920.

Gypsum Plasters for Concrete Surfaces and Gypsum Finishing Plasters: Virgil G. Marani, Permanent Builder, July, 1921.

Gypsum Tile or Block Extensively Used in Fire Resistive. Construction: Virgil G. Marani, Permanent Builder, August, 1921.

Gypsum a Structural Material: Virgil G. Marani, The American Architect, April 17, 1918.

Gipsdielen (Festigkeit, Einflusse der Feuchtigkeit, Aufaulime an Wasser, Feuersicherheit, Warmeleitung und Schalldampfung) : Tonind. Zeit., Vol. 28, pp. 908-910, 1904.

Gipsabgussen, Trankung von, zur Konservierung (Trankung mit Zapon) : Sprechsaal, Vol. 37, p. 798, 1904.

Gips als Feuerschutz-mittel: Asphalt und Teerind, Z., Vol. 7, p. 469, 1907.

Gipsverputz, Schwindrisse im.: Moye, Tonindustrie Zeitung, Vol. 32, p. 1970, 1908.

Gips Zements und farben deselben, Ziegel und Zement, pp. 790-791, 1909.

Gipses, Feuersicherheit des: Weidner, Tonindustrie Zeitung, Vol. 30, pp. 901-903, 1906.

Gipsmorteln und Estrickgips, Versuche mit: Haarman's Z., Vol. 47, pp. 45-47, 1903.

Gipsmorteln, vorbericht uber Versuche mit: Gary, Thonind., Vol. 26, pp. 984-989, 1902.

Gipsverputze, Das Auftreten von Schwindrissen im: M. Meyer, Tonindustrie Zeitung, Vol. 32, pp. 1719-1720, 1908.

Gipsserzeugnisse als Baumaterial: Baumatk, Vol. 10, pp. 5759, 1905. 
Gipsbautem in Spanien: Tonindustrie Zeitung, Vol. 31, pp. 442-443, 1907.

Gips, Bemerkungen uber den, in seiner bautechnisches Bedeutung: Annalen fur Gewerbe, Vol. 53, pp. 219-221, 1904.

Gips als feuerschutzmittel: Weidner, Tonindustrie Zeitung, p. 1180, July 20, 1912.

Gips Normen und Proben: Thonind., Vol. 24, pp. 428-430.

Gypsum $\mathrm{T}$ Beam slabs for covering roofs: Eng. News, Vol. 76, p. 1173, Dec. 21, 1916.

Gypsum Plaster and Gypsum Products as Building Materials: Pedrotti, Scientific Amer. Suppl., Vol. 64, pp. 18-19, 1907.

Gypsum Plaster on Metal Laths: Cement and Eng. News, Vol. 24, p. 215, 1912.

Gypsum Plaster on Metal Lath: S. G. Webb. Rock Products, p. 48, Apr., 1912.

Gypsum slabs for Roof Construction: Eng. News., Vol. 72, p. $246,1914$.

Gypsum Filler for Conerete Floors (tiles): Eng. News, Vol. 77, p. 173, 1917 .

Heat Insulating Materials: W. A. Hull, Proceedings Am. Soc. Testing Materials, Vol. XVII, part 2, pp. 432-442.

Houses of Gypsum. The Contract Record (Canada), Dec. 18, 1918.

La fabbricazione del gesso col sistema perin Giorn: Gen. Civ. Vol. 45, pp.'38-41, 1907.

Methods of Measuring the Time of Set of Calcined Gypsum: Emley, W. E., Am. Ceramic Soc., Vol. 19.

Manufacture of Plaster of Paris: Redgrave, Journal Franklin Institute, Vol. 10, p. 202.

Mortars, Plasters and Stuccoes, Hodgson, Fred T., F. J. Drack \& Co., Publishers, Chicago, 1906.

Platre Systeme Perine, Procede de Fabrication du: Dumas, Genie Civil, Vol. 49, p. 68, 1906.

Partition Problems in Office Buildings: Keith, Malcolm, Building Management, April, 1918.

Plasters, Floor Domes and Tile: Henning, C. F., Journal Soc. Contractors of Federal Buildings, May, 1915.

Plasters and Hard Finish Cements in the United States: Eckel; E. C., Eng. News, Vol. 49, Jan. 29, 1903. 
Plaster of Paris and the Effects of Foreign Substances: Troxell, E. R., Am. J. Science, Vol. 41, pp. 198-210, 1916.

Plaster of Paris, Shop Uses: Mack, Amer. Machinist, Vol. 31, pt. 1, p. 771, 1908.

Platre system De Coster, Installation de' usines a: Revue Ind., Vol. 34, pp. 241-242, 1903.

Platre Systeme Perin Procede de fabrication du platre et four a: Genie Civil, Vol. 46, pp. 136-139, 1904.

Plaster of Paris-Change of Volume during Setting Process: Bakker, B. A., Dental Cosmos, Vol. 58, pp. 772-780.

Reports of Committee C-11, Am. Soc. Testing Materials. Vol. XVIII, 1918, Vol. XIX, 1919, Vol. XX, 1920.

Report on Inspection of Installation of Gypsum Slab Construction for Roofs and Floors, U. S. Navy: McKay, Geo. A., Bureau of Yards and Docks Bulletin No. 25.

Regulations for Gypsum tile, Plasterboard, Roof tile, etc. National Board of Fire Underwriters, Building Code of 1915, and Dwelling House Code of 1916.

Reinforced Gypsum T Beams for Roof Decks cast at the Site and Hoisted in Place: Engineering News, Dec. 23, 1916, Vol. 74, p. 776.

Stuckgips, Bericht uber die in Munchen angestellten Versuche uber: Tonindustrie Zeitung, Vol. 30, pp. 799-804, 1906 (Festigkeit und abbindefahigkeit).

Stuckgips, totegbrannten und Estrichgips, Studien uber: Glasenapp, Tonindustrie Zeitung, Vol. 32, p. 1873, 1908.

Stuckgips, Praktisches Untersuchungsverfahren fur: Krumbbaar, Chemilker Zeitung, Vol. 32, pp. 257-258, 1908.

Stuckgips, totgebrannten und Estrichgips, Studien uber: Tonindustrie Zeitung, Vol. 32, pp. 1148-1152, 1908.

Stuckornamenten, Hertellung von: Erfind., Vol. 34, pp. 305-306, 1907 (Eingelegte Holzleisten).

Some Structural Properties of Gypsum and Reinforced Gypsum: Slater, W. A., Illinois Acadamy of Science, Transactions, Vol. 9.

Some Factors Influencing the Time of Set of Calcined Gypsum; Householder, F. S., Jour. Am. Ceramic Soc., 1918, Vol. I, No. 8.

Tests of Plain and Reinforced Conerete Specimens: Slater. 
W. A., Western Society of Engineers, Vol. XXIV, No. 7, Sept., 1919.

TESTS:

Bond Strength. On Gypsum, by the R. W. Hunt Co., Testing Engineers, St. Louis, Mo.

Corrosion. On various panels including gypsum materials by

U. S. Bureau of Standards Technologic Paper 70, 1917, Official Record.

Corrosion. Of steel in gypsum: Report of Tests of Gypsum and Reinforced Gypsum Specimens, p. 29, Jan. 18, 1917, Slater, W. A.

Corrosion. Of steel in Gypsum: U. S. Bureau of Standards, 1911, Pearson, J. C.

Crushing. On Plaster of Paris Cubes: Bureau of Standards, 1916, Griffith, J. H.

Fire. Tests of Floors in the United States: International Assn. for Testing Materials, Paper XXVII, 2, 1912.

Fire. On Metal lath and Gypsum Plaster: Records of the Associated Metal Lath Manufacturers, March 17, 1916, R. W. Hunt \& Co., Testing Engineers, Los Angeles.

Fire. On Gypsum Studs, Plaster Board and Plaster: Detroit Bldg. Dept. Official Records, June, 1904.

Fire. On Gypsum Plaster Board covered with sheet metal: Industrial Board, Dept. of Labor, New York State, Nov. 6, 1914.

Fire. On Plaster Stud, Metal Lath and Plaster: Official Records, New York City, Aug., 1905.

Fire. On Gypsum Plaster Board: Atchison, Kansas, Board of Education, April 10, 1908.

Fire. On Gypsum Plaster board: Associated Mutual Fire Ins. Co.

Fire. On Gypsum Plaster Block: British Fire Prevention Committee, 1899, 1900, 1903, 1904.

Fire. On Gypsum Plaster Board: Berlin Metropolitan Fire Brigade, Report of 1900.

Fire. On Gypsum Plaster Board and other Material: Official Records, Institute of Technology, Feb. 3, 1900, Prof. C. L. Norton. 
Fire. On Gypsum Plaster Board by Underwriters' Laboratories, Inc., Chicago, Report No. 336.

Fire. On Gypsum Wall Board by Underwriters' Laboratories, Ine., Chicago, Report No. 1006.

Fire and Load. On Gypsum Floor: Columbia University for City of Toronto, Toronto Bldg. Dept., and Gypsum Industries Association Records, Aug., 1913.

Fire and Load. On Gypsum Floor, 1897: Columbia University for New York City, Official Records.

Fire-and Strength. On Gypsum Plaster Board, by Kansas City Board of Appeals, June 14, 1915.

Fire and Water. On Gypsum Tile: New York City, Nov. 6, 1903, Aug. and Oct., 1901, June, July and Nov., 1905, Aug., 1906, Nov., 1908, Official Records.

Fire and Water. On Metal Lath and Plaster: New York City, Aug. and Oct., 1901, Official Records.

Fire and Water. On Gypsum Plaster Tile: Philadelphia Bldg. Dept., March, 1905, Official Records.

Fire and Water. On Gypsum Plaster Board and Plaster: Portland, Oregon, Bldg. Dept., June 9, 1911, Official Record. Fire and Water. On gypsum partition tile, by Underwriters' Laboratories, Inc., Chicago, Aug., 1909, Official Records, Rept. No. 378.

Fire and Water. On gypsum warehouse partitions, by Underwriters' Laboratories, Inc., Chicago, Dec. 13, 1917, Official Records.

Fire and Water. On gypsum partitions for storage warehouses (not plastered) by Underwriters' Laboratories, Fnc., Chicago, Jan. 31, 1918.

Fire and Water. On gypsum tile, by U. S. Bureau of Standards, Pittsburgh, Pa., Dec. 19, 1913, Official Records.

Fire and Water. On gypsum plaster board and plaster, by U. S. Gypsum Có., Chicago, July 19, 1913.

Fire, Load and Water. On gypsum and other floors: International Assn. for Testing Materials, Sixth Congress, Paper XXVIT.

Fire, Load and Water. On gypsum floor. New York City, May 20, 1897, Official Records. 
Fire, Load and Water. On gypsum floor: Phila. Building Dept., Sept., 1912, Official Records.

Fire, Load and Water. Tests on gypsum floors by Jas. S. McGregor, Columbia University, 1897, 1912, 1913, Official Records.

Fire, Load and Water. On gypsum stairway and elevator enclosures, by. Underwriters' Laboratories, Chicago, Feb. 1, 12, 1918, Official Record.

Fire, Water and Strength. On gypsum tile, by Underwriters' Laboratories, Inc., Chicago, June 22, 1910, Official Records.

Flexure, Soaking and Freezing. On gypsum roof tile: Columbia University, Jan. and Feb., 1917.

Heat Conductivity. On gypsum and other materials: New York City, Nov. 6, 1903, Official Records. Files of U. S. Gypsum Co. Heat Conductivity of gypsum roof tile, Investigations by Prof. R. C. Carpenter, Cornell University, 1916-17. Heat Insulating Properties of gypsum and other materials. W. A. Hull, Bureau of Standards, Pittsburgh (See American Society for Testing Materials Proceedings, Vol. 17, Part 2, 1917. Also American. Concrete Institute, Vol. 2, No. 6, 1914).

Heat Insulating Properties. Published in American Society for Testing Materials Proceedings, Vol. XVII, Part 2, 1917, Bureau of Standards, Pittsburgh, W. A. Hull: also American Concrete Institute, Vol. II, No. 6, 1914.

Heat Insulation. On gypsum and other material: Massachusetts Inst. of Technology, 1914, Official Records, Prof. C. L. Norton.

Loading Test. On gypsum slab: Bureau of Standards, Nov. 14, 1918. Reported in Bulletin 25, U. S. Navy (Bureau of Yards \& Docks), Official Records.

Loading Test. Of gypsum composition floor: Eng. and Contracting, Vol. 47, pp. 82-85, Jan., 1917. U. S. Bureau of Standards.

Impact. On gypsum tile partitions and clay tile plastered, by U. S. Gypsum Co., to Lewis Institute, Chicago, No date. Report of Committee C-11 on Gypsum, Am. Society for Testing Materials Proceedings, Vol. XIX, 1919. 
Report on Tests of plain and reinforced gypsum specimens; Western Soc. of Engineers: W. A. Slater and G. P. Anthes (forthcoming paper, Oct. 13, 1919).

Report on Structural Properties of gypsum and reinforced gypsum: Illinois Acad. of Science Transactions, Vol. 9, W. A. Slater.

Thermal Conductivity. Armour Inst. of Technology, March 28, 1914, also 1916 and 1918.

Sound. On gypsum tile and clay tile plastered, by Prof. Phillip B. Woodworth, Lewis Inst., Chicago, May 19, 1914.

Sound. On gypsum tile, plaster board and other materials plastered, by Dr. F. R. Watson, University of Illinois, 1916-1917.

Sound. On gypsum plaster board plastered, clay tile and gypsum tile, by Prof. Philip B. Woodworth, Lewis Inst., Chicago, June 3, 1914.

Sound Transmission. On gypsum tile, plaster board and other materials: Lewis Inst., Chicago, 1914.

Sound Transmission. On gypsum plaster board and other materials: Prof. C. L. Norton, Massachusetts Inst. of Tech., March 16, 1902, Official 'Records.

Steam, Hot Gases, Hot Water, Acids. On gypsum roof tile, by E. T. DuPont DeNemours \& Co., May 17, July 17 and 31, 1917.

Strength. On suspended ceilings of gypsum plaster board and plaster: Philadelphia, Nov., 1916. Pains, McLennan \& Campion, Engineers.

Strength of gypsum floor, for Philadelphia by Long \& Miller, New York, May 29, 1912.

Strength Tests. Flexure tests on reinforced gypsum roof tile, by James S. MacGregor, Columbia University, New York, Jan., Feb., 1917.

Strength. On gypsum floors, by Structural Engineering Co., New York, Apr. 27, 1896, Official Records.

Strength. On reinforced and plain gypsum specimens, by W. A. Slater, Tllinois University, 1914, 1915, 1916, 1917.

Strength. On many makes of wall boards, Underwriters' Laboratories, Chicago, Ill., 1918, Official Record, Manufacturers files and G. I. Association. 
Strength. On gypsum roof tiles, by Prof. R. H. Danforth, Case School of Applied Science, Cleveland, Ohio, 1917.

Strength. On gypsum roof tile, by M. O. Withey, Univ. of Wisconsin, 1916.

Strength. On gypsum plaster and wall boards, by Underwriters' Laboratories, Inc., Chicago (report to War Industries Brd. and War Service Committee on Gypsum) 1918.

Strength of Concrete when poured in contact with gypsum. R. W. Hunt Co., Testing Engineers, Chicago, March 27 and June 21, 1916.

Sulphur, Ammonia, Steam.Tests on gypsum roof tile by Milwaukee Coke \& Gas Co., Sept. 10, 1915.

Sulphur Fumes, Etc. On gypsum tile, by A. S. Ross, Chemist, Milwaukee Coke \& Gas Co., Sept. 10, 1915.

Underwriters' Laboratories, Inc., Chicago (Fire test on plaster block), Aug., 1909, Report 378.

Underwriters' Laboratories, Inc., Chicago (Fire test on plaster board), Aug., 1909; Report 336.

Underwriters' Laboratories, Inc. (Fire test to determine rate of calcination of gypsum), June 2, 1912; Official Records.

Underwriters' Laboratories, Inc. (Fire test on gypsum tile for warehouse partitions), Dec. 13, 1917, Official Records.

Underwriters' Laboratories, Inc. (Fire test on gypsum stairway and elevator enclosures), Feb. 1, 12, 1918.

Underwriters' Laboratories, Inc. (Fire test on gypsum tile for warehouse partitions, not plastered), Jan. 31, 1918.

Underwriters' Laboratories, Inc. (Fire test on column protection materials including gypsum tile), July, 1918.

Underwriters' Laboratories, Inc. (Fire and Water test on wall board), July 20, 1919, Report 1006.

Underwriters' Laboratories, Inc. (Fire and water .test on plaster block), June 22, 1910, Official Records.

Underwriters' Laboratories, Inc. (Strength tests on gypsum wall board), 1918, Report of War Industries Board and War Service Committee on Gypsum.

United' States Bureau of Standards, Pittsburgh, Pa. (Fire and water test on gypsum tile for City of Pittsburgh), Dec. 19, 1913, Official Records. 


\section{HYDRAULIC GYPSUM}

Anhydrit Zen Herstellung von Estrichgips: Tonindustrie Zeitung, pp. 749-751, 1909.

Des Hydraulic, Estrich Gips, Van't Hoff und Just, 1903.

Estrichgips, Herstellung von: Tonindustrie Zeitung, Vol. 28, pp. 709-710, 1904.

Estrichgips, Asphalt und Terind. Zeit., Vol. 7, pp. 505-506, 1907.

Estrichgips und Gipsmorteln, Versuch mit: Gary, Mitteilungen Versuch, Vol. 20, pp. 1-40, 1902.

Listrichoips und Gipsmorteln, Versuch mit: Haarmann's Zeit., Vol. 47, pp. 45-47, 1903.

Estrichgips, etc., Studien uber: Rohland, Tonindustrie Zeitung, Vol. 32, p. 1873, 1908.

Estrichgips, etc., Studien uber: Glasenapp, Tonindustrie Zeitung, Vol. 32, pp. 1148-1152, 1908.

Estrichgips (Anwendung, Prufung): Tonindustrie Zeitung, Vol. 28, pp. 668-672, 1904.

Estrichgips, studien uber, etc.: M. Glasenapp, Regasche Industrie Zeitung, pp. 177, 193, 209, 1909.

Gipsestrichboden, Herstellung von: Erfind, Vol. 34, pp. 602604, 1907.

Gipsestrich, Bericht des Ausshusses Zun Verarheitung Von: Cramer, Tonindustrie Zeitung, Vol. 29, pp. 791-793, 1905.

Gipsestrich, Erprobung von (Bericht): Tonindustrie Zeitung, Vol. 27, pp. 454-455, 1903.

Gipses, Hydraulischen, vergrosserung des Abbindevermogens (Behandlung mit Kalisalzen) : Erfind, Vol. 34, pp. 33-34, 1907. Hydraulic Gypsum (Estrich): Thonind., Vol. 25, p. 1581.

The Manufacture of Hydraulic Gypsum: Glasenapp, Cement and Eng. News, Vol. 22, pp. 51, 95, 145, 1910.

Uber Hydraulischen Gips, Mack, 1896.

Uber den Estrichgips: O. Frey, Tonindustrie Zeitung, Vol. 34, pp. 170-172.

\section{GYPSUM IN PORTLAND CEMENT}

Effect of Finer Grinding and a Higher $\mathrm{SO}_{3}$ Content upon the Physical Properties of Portland Cement: P. H. Bates, Am. Soc. for Testing Materials Proceedings, 1915. 
Gypsum in Portland Cement,-Permissible amounts, and Methods of Analysis: Am. Soc. for Testing Materials, 1916.

Gypsum as an Addition to Cement: S. Stein, Thon. Ind., Vol. 40, p. 140, 1916-Reviewed in Chem. Abst., Vol. 10, No. 22, p. $2974,1910$.

Gipses, Wirkung des, auf Portlandzement: Moye, Tonindustrie Zeitung, Vol. 32, p. 117, 1908.

Gips und Portland Zement: H. Schafer, Moye, Zem. u Bet., Vol. 5, pp. 325-328, 348, 1906.

Zement, Zusetsen von, mit Gips: Haarmann's Zeit., Vol. 46, pp. 103-104, 1902.

Zement, das Zusetsen von, Zun Gips: Schwalm, Thonind., Vol. 26, p. 393, 1902.

\section{MISCELLANEOUS}

Artificial stone from gypsum: Builder, Sept. 26, 1913.

Directory of Portland Cement, Gypsum \& Lime Manufacturers, 1912-1921.

Ein Plastischer Gyps mortel aus alter Zeit: Haarman's Zeit., Vol. 44, pp. 62-63.

Fabrication de corps en platre Hydraulique a prise rapide: Mack, Mon. Cer. Vol. 27, p. 171.

Gips, Uber, (Physikalische Vorgange bei der Erhartung): Port, Mon. Zahn., Vol. 23, pp. 531-538, 1905.

Gipsabgusse, Wiederherstellung unansehnlich gewardener (Milch, Kalkwasser und Leim): Frfind, Vol. 34, pp. 247249, 1907.

Gips Widerstandsfahig Machen: Met. 'Techn., Vol. 33, p. 397, 1907.

Gipsgussformen, Sehmiermittel Zam Anstreichen von (Fein Zerteilte Stearinsaure, damit sich kein staub ansetzt): Z. Drechsler, Vol. 30, p. 111, 1907.

Gipses, Zur Erhartung des: Rohland, Tonindustrie Zeitung, Vol. 30, pp. 1656-1657, 1906.

Gips und Gipsgiessen (Harten, Giessen, Verwertung alter Gipsformen) : Sprechsaal, Vol. 36, pp. 1882-1884, 1903.

Gips, Farben von, Verfahren Zum, Vanino; Erfind., Vol. 28, pp. 110-111, 1901. 
Gypsum and its Use in Dentistry: Moser, J. Zahn, Vol. 13, Nu. 15.

Gypsabgussen, Trennung von: Port, Corresp. Zahnheilk, Vol. 35, pp. 133-134, 1906.

Gips, (Untersuchungen aus): Krumbhaar, Tonindustrie Zeitung, Vol. 30, pp. 2173-2176, 1906.

Gips, Neues aus dem auslandischen Schriften uber: Klehe, Tonindustrie Zeitung, Vol. 30, pp. 953-961, 1906.

Gipsgusse, Blendend weisse und glanzende (Leimlosung dann Glanzpickfarbe) : Reg. Ind. Z., Vol. 33, pp. 199-200, 1907.

Gips und seine Verwendung in der Farben-Fabrikation; Farben Z., Vol. 10, pp. 1-21, 1904.

Gipses, Kontrahieren des (Verringerung durch Auruhren mit Kalkwasser) : Dodel, J. Zahnheilk., Vol. 19, p. 13, 1904.

Gypsum Product for Coating Paper. J. Soc. Chem. Ind., Vol. 35, p. 839, 1916.

Gypsum Powder for Coating Paper. Chem. Abstract, Vol. 10, p. 1595, 1916.

Gypsum, Properties, Definitions and Uses: S. W. Stratton, Bureau of Standards Circular 108, 1921.

Gips, Verfahren Zur Herstellung von zun Fuller Papier U. dgl. geeignetem: Erfind., Vol. 33, pp. 268-269, 1906.

Hardening of Gypsum for Electrical Purposes: Thonind., Vol. 25, pp. 12-13.

Harten Des Gypses, Pharm. Centralb., Vol. 41, p. 576.

H.arten von Gips. Gen. Bl. Wurt., Vol. 52, p. 76.

Harten von Gips; Apoth. Zeit., Vol. 15, pp. 819-820.

How Plaster of Paris is Utilized for Patterns: R. R. McGowan, Foundry, Jan., 1914, pp. 24-28.

Monographie hygienique de la fabrication du platre en France: A. Hebut and F. Heine, 51 pp., 1901.

Nouveau traitement du gypse paru produire du marbre artificiel: Packer, Mon. Ser., Vol. 28, p. 207.

Normal Consistency of Sanded Gypsum Plaster: Emley and Faxon, Jour. Am. Cer. Soc., Feb., 1921.

Present and Future of the American Gypsum Industry: Frank A. Wilder, Eng. and Min. Jour., Aug. 30, 1902.

Plaster of Paris, Use of, in the Foundry : Buchanan, Mechanical World, Vol. 41, p. 134; Foundry, Vol. 29, pp. 403-404, 1907. 
Plaster of Paris in the Pattern Shop: Amer. Machinist, Vol. 26, p. 1119, 1903.

Plaster Patterns: Mechanical World, Mar. 22, 1912.

Process of Briquetting Ores before Smelting (using gypsum and pitch): O. Bolton, U. S. Patent 1166171, 1915.

Solutions for Hardening Gypsum: Haarman's Zeit., Vol. 45, p. 192.

The Hardening of Gypsum: Zulkowski, Chem. Ind., Vol. 22, pp. 349-352.

Verwerthung Alter Gypsformer: Thonind., Vol. 21, p. 901. Uber das Abbinden und Abharten Gypses: Thonind., Vol. 19, p. 29.

\section{AGRICULTURAL GYPSUM}

OLDER REFERENCES

Baxter, Library of Practical Agriculture, Vol. 2, pp. 45, 58, Sussex Agrl. Press, London, 1846.

Binns, John A., A treatise on practical farming embracing particularly the following subjects, viz; The use of Plaster of Paris, with Directions for using it; and general Observations on the Use of the other Manures. On Deep Plowing; Thick sowing of Grain; Method of Preventing Fruit Trees from Decaying and Farming in General, Published by John B. Colvin, Fredericktown, Md., 1803.

Fothergill, A., M. D., F. R. S., etc., Essays on the Fertilizing Effects of Gypsum in the United States of America; with a view to Elucidate its mysterious Operation, and to Extend its Benefits to England and other parts of the British Dominions, Communications to the Board of Agriculture of Great Britian, 6, 370-380, 1808.

Davey, Sir Humphrey., Elements of Agricultural Chemistry; Longman, Hurst, Rees, Orme and Brown, London, 1813.

Kirwin, Richard, The Manures most Advantageously Applicable to the Various Sort of Soils and the Causes of their Beneficial Effects in each Particular Instance, 6th Ed. London, 1806, Gypsum pp. 90-91.

Johnson, Cuthbert William., An account of the Application of Gypsum as Manure to Artificial Grasses. A Prize 
. Essay, Roy. Agr. Soc. Jour., 2; 106-112, 1841. (This is the same article that appears in his Encyclopedia, 1842). Johnson, Cuthbert William, The Farmers Encyclopedia and Dictionary of Rural Affairs embracing all the most recent discoveries in Agricultural Chemistry adapted to the Comprehension of unscientific Readers. Longman, Brown, Green and Longman, London, 1842.

Loudon, J. C., An Encyclopedia of Agriculture, Longman, Orme, Brown, Green and Longmans, London, 1839.

Mr. Smithe of Tunstall, near Sitting Bourne; Kent, A brief Reply to the several undermentioned Queries, proposed by Sir John Sinclair, respecting the Application and Effect of Gypsum as a Manure for Artificial Grasses, etc., Communications to the Board of Agriculture of Great Britain, 6, 262-267, 1808.

Nicholson, John, The Farmer's Assistant, Herkins County, New York, pp. 1.33-135, 1814.

Peters, Judge Richard, of Philadelphia, A Treatise on Land Plaster, prepared at the request of General Washington, January, 1797, Revised for presentation to their agricultural friends by Smith and Harris, Coates St. Wharf, Phila.

Peters, Judge Richard, Agricultural Inquiries on Plaster of Paris, Also Facts, Observations and Conjectures on that Substance, when applied as manure, collected chiefly from the practice of farmers in Pennsylvania, and published as much with a view to invite as to give information. Charles : Cist and John Markland, Philadelphia, 1797. Entered according to Act of Congress.

Washington, Geo., Letters from his Fxcellency George Washington to Arthur Young, Esq., F. R. S., and Sir John Sinclair Bart., M. P., containing an account of his husbandry with his opinion on various questions in agriculture and many particulars of rural economy of the United States, Gotton and Stewart, Alexandria, 1803. Gypsum pp. 122-123. 
Calcium Sulphate in Aqueous Solutions: Cameron and Bell, U. S. Department of Agriculture, 1906.

Cramer: Dungegips, Tonindustrie Zeitung, Vol. 29, pp. 780-782, 1905.

Headden, Wm. P., Black Alkali in the San Luis Valley: Colorado Agrl. College Bull. 231, Oct., 1917.

Hilgard, E. W., Soils, Published by MacMillan Co., p. 458, (Gypsum and Black Alkali) 1906.

Hilgard, E. W., Alkali, Its Nature, Cause and Effect, Rept. Agrl. Expt. Station, Univ. of Colifornia, 1890.

Merz, Ph., Der gips in den Lands-withschaft als Konservierengs und Dunge mittel, Verlag Tonindustrie-Ztg., Berlin, 1903. (Translated by Southern Gypsum Co., Inc., North Holston, Va.)

Soavo, Marco, Intorno al meccanesimo di azione del gesso impiegato in agricoltura: Staz. sper. agrar. Ital., 41, 473-506, 1908. (A full review of the action on the soil of gypsum alone, of gypsum mixed with blood, and with $\left(\mathrm{NH}_{4}\right)_{2} \mathrm{SO}_{4}$, under possible practical conditions. Gives short list of books referred to.)

Plaster or Sulphate of Lime: The Southern Planter and Farmer, March, 1878.

The use of Plaster as it Affects the Physical Condition of the Soil: The Southern Planter and Farmer, March, 1876.

Gyps als Dungemittel: Thonind., Vol. 24, pp. 154-155.

Reclamation of Alkali Lands with Gypsum at the Tulare (California) Experiment Station: Rpt. Agrl. Exp. Sta. Univ. of California, 1892-93.

Il gesso in agricolturo: Bonacino, A:, Ann. R. Ac. Agr. Tormin, $50,1907$.

Gips als mittel gegen den Getreiderrost: Stein und Mortel, Vol. 12, pp. 328-329, 1908. 
BIBLIOGRAPHY OF GEO. H. OLSEN AND J. L. ST. JOHN*

(1) Ames, J. W., and Boltz, G. E., Effect of Sulfofication 1919 and Nitrification on Potassium and Other Soil Constituents: Soil Sci., Vol. $7: 3$, pp. 183-196.

(2) Ames, J. W., and Boltz, G. E., Sulfur in Relation to 1916 Soils and Crops: Ohio Agr. Exp. Sta., Bul. 292, pp. 219-256.

(3) Ames, J. W., and Richmond, T. E., Effect of Sulfofica1918 tion and Nitrification on Rock Phosphate: Soil Sci., Vol. 6:3, pp. 351-64.

(4) Ames, J. W., and Richmond, T. E., Fermentation of 1917 Manure Treated with Sulfur and Sulfates. Changes in Nitrogen and Phosphorus Content: Soil Sci., Vol 4, pp. 79-89.

(5) Ames, J. W., and Richmond, T. E., Sulfofication in 1918 Relation to Nitrogen Transformations: Soil Sci., Tol. 4, pp. 311-321.

(6) Andre, G., Deplacement de la Potasse Continue Dans1913 certaines Roches, Feldpathiques par Quelques Substances Employess Comme Engrais: Compt. Rend. Acad. Sci. (Paris), $157: 19$, pp. 856-58.

(7) BanBussy., Jour. Pharm., 27, 39; Ann., 34, 223

(8) Barger and Ewin, Jour. Chem. Soc., Vol. 99, p. 19112336.

(9) Batten, E. T., Peanut Culture: Virginia Agr. Exp. 1918 Sta. Bul. 218, pp. 1-16.

(10) Bear, Firman E., and Workman, Albert C., The Am1919 monia-Fixing Capacity of Calcium Sulfate: Soil Sci., Vol. $7: 4$, pp. 283-293.

(11) Bernhard, A. D., Action of Sulfur as a Fertilizer. 1911 Deut. Landw. Presse, Vol. 39, p. 275; Chem. Abst., 7, 530 .

(12) Bernhard, A. D., Experiments on the Control of Potato 1910 Scab: Deut. Landw. Presse, $37: 18$, p. 204; Exp. Sta. Rec. 23, 744.

(13) Bernliard, A. D., Sulfur as a Fertilizer: Deut. Landw. 1912 Presse, 39, p. 275; Exp. Sta. Rec. 27, p. 422.

*In "An Investigation of Sulphur as a Plant Food" State College of Washington Agri. 'Experiment Station Bull. 165, May, 1920. 
(14) Bernhard, A. D., Chem. Abst., $7: 6$, p. 1071.

(15) Berry, R. A., The Results of Some Experiments With 1914 Barnyard Manure: West of Scotland Agr. Col. Bul. 65.

(16) Bjorn, Anderson H., The Loss of Nitrogen from Liquid 1905 Manure and Its Prevention by Super-Phosphate: Tdsckr. Landolonom, No. 3, pp. 160168.

(17) Blair, A. W., Potash and Crops: New Jersey Circ. 191767.

(18) Bogdanov, S., On the Sulfur in Plants: Zur. Russ. Fiz. 1899 Kin. Obshch, 31:4, p. 471; Exp. Sta. Rec., 11, p. 723.

(19) Bohme, E., Preservation and Action of the Nitrogen of 1905 Urine: Centbl. Agr. Chem. Bul. 34, No. 5, p. 300.

(20) Boiret, H., and Paturel, G., Use of Iron Sulfate in 1892 Agriculture: Ann. Agron., 18, pp. 417-40; Exp. Sta. Rec., 4, pp. 435, 436.

(21) Bossinelli, C., Intorno Alla Azione Dello Zolfo Libero 1915 Sulla Vegetazione: Staz. Sper. Agr. Ital., Vol. 48, No. 3, pp. 175-184.

(22) Boullanger, E., Etudes Experimentales sur la Engrais 1912 Cataliques: Ann. Sci. Agron., t. 4, ser. 1, No. 3, pp. 161-180.

(23) Boullanger, E., L'action des Soufre en fleur sur la Vegetazione: Compt. Rend. Acad. Sci. (Paris) t. 154: 6, pp. 369, 370; Chem. Abst. 6:10, 1332.

(24) Boullanger, E., and Dugardin, M., Mecanisme fe l'action Fertilisante du Soufre: Compt. Rend. Acad. Sci., t. 155:4, pp. 327-329.

(25) Bowker, American Fertilizer, Aug. 26, 1911. 1911

(26) Bradley, C. F., Calcium Oxide and Calcium Sulfate in 1910 Oregon: Jour. of Industrial and Engr. Chem., Vol. 2, pp. 529, 530.

(27) Bradley, C. E., Soils in Oregon: Oregon Agr. Exp. 1912 Sta.. Bul. 170.

(28) Briggs, L. J., and Breazeale, J. F., Availability of Pot- 
1917 ash in Certain Orthoclase-bearing Soils as Affected by Lime or Gypsum: Jour. Agr. Res., Vol. 8, pp. 21-28.

(29) Brioux and Guerbet., Oxidation of Sulfur Is Bacterial: 1913 Compt. Rend., 156, p. 2476.

(30) Brodie, O. A., Manure: U. S. Dept. Agr. Farmer's 1918 Bul. 978.

(31) Brooks, W. P., and Garkell, E. F., Fertilizer Experi1919 ments: Massachusetts Sta. Report, Vol. 9, p. 339.

(32) Brioux, Ch., and Guerbet, M., Ann. Cci. Agr., 30, 38998, Chem. Abst., 8:9, 1481; Compt. Rend., $156: 91,1476$; Exp. Sta. Rec., 2.

(33) Brown, P. E., The Fertility of Towa Soils: Iowa Exp. 1914 Sta. Bul. 150.

(34) Brown, P. E., and Gwinn, A. R., The Effect of Sulfur 1917 and Manure on Availability of Rock Phosphate in Soil. Towa Exp. Sta. Res. Bul. 43.

(35) Brown, P. E., and Johnson, H. W., Studies in Sulfofi1916 cation: Iowa State College Agr. Exp. Sta. Bul. 34.

(36) Brown, P. E., and Johnson, H. W., Studies in Sulfo. 1916 fication: Soil Sci., Vol. 1, pp. 339-62.

(37) Brown, P. F.', and Kellogg, E. H., Sulfur and Permanent Soil Fertility in Iowa: Jour. Amer. Soc. Agron., Vol. 7, 97-108.

(38) Brown, P. E., and Kellogg, E. H., Sulfofication in 1914 Soils: Res. Bul. 18, Iowa State College.

(39) Brown, P. E., and Kellogg, E. H., Sulfofication in Soils: Proc. Iowa Acad. of Sci. 21, 17.

(39a) Brown and Kellogg. Determinations of Sulfur in 1915 Soils: Jour. Ind. and Eng. Chem., Vol. 7, p. 686.

(40) Brown, P. E., and Warner, H. W., The Production of 1917 Available Phosphorus from Rock-phosphate by Composting with Sulfur and Manure: Soil. Sci., Vol. 4:4, pp. 269-82.

(41) Burt, B. C., and Leather, J. W., Amount and Composi1909 tion of Drainage Water at Cawnpore Agr. Exp. Sta.: Cawnpore Agr. Exp. Sta. Report, 1909, pp. 23-29. 
(42) Byer. Concerning the Influence of Salt Solutions and 1871 Other Weathering Agencies on the Decomposition of Feldspars: Jahresber. Agr. Chem., Bd. 13, p. 22.

(43) Cameron, F. K., and Patten, H. E., The Distribution 1907 of Solute Between Water and Soil: Jour. Phys. Chem., Vol. 11, p. 581.

(44) Carlier, A., The Value of Magnesium Sulphate Plus 1910 Manganese Sulphate as a Fertilizer: Jour. Agr. Prat., 1:267; Chem. 'Abst. 5:16, 2687; Engrais, 25, 888; Chem. Abst., 5:18. 3116.

(45) Cercelet, M., Catalytic Fertilizers: Rev. Vit., 38, 381; Chem. Abst., 7, pp. 529.

(46) Cettoline, Sante, Experimenti di Concimanzione con 1915 Sostanze Complimentari (Catolitici) della Cencere della Vita: Bol. Quind. Soc. Agr. Ital., Vol. 20, No. 13, pp. 431-38.

(47) Chancrin and Desriot., Action of Sulfur as a Fertilizer: 1909 Jour. Agr. Prat., 21 :427; Chem. Abst., 6, 789.

(48) Chancrin and Desriot., Fertilizing Action of Sulfur: Jour. Agr. Prat. n. ser., 25, 364; Chem. Abst., $7: 32,4038$.

(49) Chancrin and Desriot, Influence of Sulfur on the Growth of Potatoes and Beets:Jour. Agr. Prat., n. ser., 21:14, pp. 427-429; Chem. Abst., $7: 23$, 4038.

(50) Chauzit, Jean, Fertilizing Action of Sulfur on Vines: 1914 Rev. Vit., 41, pp. 175-9; Chem. Abst., 8, 1482.

(50a) Christie, A. W., and Martin, J. C., The Volumetric De1917 termination of Sulfates in Water Extracts of Soils. Soil Science, Vol. $4: 6$.

(51) Clark, F. W., Data of Geochemistry: U. S. Geol. Sur1908 vey Bul.. 330, p. 26.

(52) Collison, Stanley E., and Walter, Seth S., Loss of 1916 Fertilizers by Leaching: Florida Agr. Exp. Sta. Bul. 132, pp. 1-20.

(53) Conn, Agr. Bacteriology, p. 59, An Account of Sulfur Bacteria.

(54) Copenhagen Station Results. The Storage of Liquid 
1906 Manure: Abs. in Exp. Sta. Rec., Vol. 5:8, p. 618.

(55) Creldt, von Seelhorst, and Wilms, Untersuchungen 1901. uber Drainage Wasser: Jour. Landw., Vol. 49, pp. 251-275.

(56) Crocker, History of the Use of Gypsum as a Fertilizer, Univ. of Chicago Paper.

(57) Curry, B. E., and Smith, T. O., Granitic Soil Potas1914 sium and Its Relation to the Production of Hay: New Hampshire Agr. Exp. Sta. Bul. 170.

(58) Daikuhara, G., Are Soils Containing Less than .02 1907 per cent Sulfur-Trioxide Benefited by Special Manure with Sulphates?: Bul. Imp. Cent. Agr. Exp. Sta. Japan, 1:2, pp. 135-143; Exp. Sta. Rec. $19: 11 ; 1022$.

(59) Darnell, Smith, Report Go't. Bur Microbiol., New South Wales, Vol. 2, p. 209.

(60) Degrully, L., The Fertilizing, Action of Sulfur: Prog. Agr. et Vit., 33, 321-24; Chem. Abst., 6:12, 1649 ; Exp. Sta. Rec., 27, 422.

(61) Deherain, P. P., Drainage des Terres Nues, Traite 1902 de Chimie Agricule, pp. 586-587.

(62) Deherain, P. P., Sur la Production des Nitrates dans la Terre Arable: Ann. Agron., t. 13, pp. 241261.

(63) Deherain, P. P., and Dupont, C., On thr Fermentation 1901 of the Nitrogenous Matter of Manure: Ann. Agron., t. 27, No. 9, pp. 401-427.

(64) Demolon, A., Recherches sur L'action Fertilisante du 1912-13 Soufre: Compt. Rend. Acad. Sci., t. 156, No. 9, 725-728; 154, No. 8, 524-26.

(65) Dezani, Action of Gypsum on Nitrification: Staz. 1911 Sper. Agr. Ital., Vol. 44, No. 2, pp. 119-137: Abst. Jour. Chem. Soc. London, Vol. 100, pt. 2, p. 1019.

(66) Dietrich, Arch. Pharm., 24.0, 161. 1902

(67) Dietzall, B. E., Experiments on the Preservation of 
1897 Barnyard Manure: Landw. Vers. Sta., Bd. 48, No. 3:5, p. 163.

(68) Duley, F. L., 'The Relation of Sulfur in Plant Materials 1916 and their Variations with the Soil Supply: Jour. Amer. Soc. Agron., Vol. 8:3, pp. 154-160.

(69) Dumont, J., Action des Composes Caliques sur la Mib1904 ilisation de la Potasse du Sol: Bul. Soc. Nat. Agr. France, t. 64, No. 5, pp. 379-384.

(70) Dumont, J., The Fertilizing Value of Plaster: Sci. 1907 Agron., Vol. 2:4, pp. 257-274.

(71) Dusserre, C., Tests of the Use of Gypsum as a Fertili1908 zer: Ann. Agr. Suisse, t. 9, No. 1, pp. 7-9.

(72) Dyer, Bernard, A Chemical Study of Phosphoric Acid 1901 and Potash in Wheat Soils of Broadbalk Field, Rothamsted: Phil. Trans. Royal Soc. Bul. 194, pp. $235-290$.

-(73) Dyer, Bernard, On the Analytical Determination of 1894 Probably Available Mineral Plant Food in Salts: Jour. Chem. Soc., 65, p. 148.

(74) Dymond, Hughes and Jupe, Jour. Agr. Sci., Vol. 1, p. 217.

(75) Ebermayer, E., Untersuchungen Uber die Sicker1890 wassermenger in Vershuden Bodenarten: Forsh. Geb. Agr. Phys., 13, pp. 1-15.

(76) Echart, C. F., Lysimeter Experiments: Hawaiian Sug1906 ar Planters' Assn. Exp. Sta. Agr. and Chem. Bul., 19, pp. 1-31.

(77) Ellett, W. B., and Harris, W. G., Co-operative Experi1920 ments for the Composting of Phosphate Rock and Sulfur: Soil Sci., Vol 10:4, pp. 315-327.

(78) Engberding, D., Verleichende Untersuchungen Uber 1909 die Bakterienzahl im Askerboden in, Ihrer Abhangigkeit von Ausseren Einflussen; Centbl. Bakt., Abt. 2, Bd. 23, pp. 569-642.

(79) Euler, Assimilation der Mineralstoff, Ergenbruisse 1909 Pflanzenchemie, Parts II and III, p. 144.

(80) 'Suler, Schwefelhaltige Pflanzenstoffe, Grundlangen and 1908 Ergébruisse Pflanzenchemie, Part I, p. 203.

(81) Feilitzen, H., One Use of Sulfur for Prevention of 
1913 Potato Scab: K. Landtbr. Akad. Handl. och Tidskr., 52:2, pp. 120-30. Indirect Fertilizer. Exp. Sta. Rec., 30-2, p. 139.

(81) Feilitzen, H., Uber die Verwendung der Schwefelblute 1913 zur Bekampfung des Kartoffelschorfes und, als indirekes Dungemittel: Fuhling's Landw. Ztg., Bd. 62, pp. 231-242.

(83) Fellers, C. R., The Effect of Inoculation, Fertilizer 1918 Treatment and Certain Minerals on the Yield, Composition and Nodule Formation of Soybeans: Soil Sci., Vol. 6:2, p. 113.

(84) Fourcray and Tingry, Crells. Anal., 2, 38, 68, 136, 1790. 1799

(85) Fraps, G. S., The Determination of Sulfur in Plants: 1901-03 North Carolina Ann. Report, pp. 42-47.

(86) Fraps, G. S., The Effect of Additions upon the Avail1916 ability of Soil Potash, and the Preparation of Sugar Humus: Texas Agr. Exp. Sta. Bul. 190, p. 30 .

(87) Fred, E. B., and Hart, E. B., The Comparative Effect 1915 of Phosphates and Sulphates on Soil Bacteria: Wisconsin Agr. Exp. Sta., Res. Bul. 35.

(88) Galstev and Yakushkin, Isv Moskov Selsk Khoz Inst. 1913. Ann. Inst. Agron. Moscow, Vol. 19:1, pp. 223-32.

(89) Gardner, Frank D., Nolls, Charles F., and Baker, P. S., 1917 Summary of the Results Obtained from Experiments with Commercial Fertilizers, Barnyard Manure, Lime and Land Plaster for a Period of thirty-five Years: Pennsylvania Agr. Exp. Sta. Bul. 146.

(90) Gerlach, The Action of Gaseous Ammonia on Super1916 Phosphates: Z. Angew Chem., Vol. 29, p. 134; Chem. Abst., Vol. 10, No. 8, p. 1071.

(91) Giannetto, F., Sulfur as a Fertilizer: Bol. Quind. Soc. Agr. Ital., 17, 425; Chem. Abst., 7:7, 1254.

(92) Gilbert, J. H., The Rothamsted Experiments: Trans. 1895 Highland, and Agr. Soc., Scotland. 55:7, 254.

(93) Gilbert, J. H., Rainfall: Evaporation and Percolation: 1876. Rothamsted Memoirs, Vol. 5:5, pp. 1-13. 
(94) Godamer, Ber., 30, 2332; Chem. Zentr., II, 922, Arch. 1897

(95) Goessmann, Chas. H., Haskins, H. D., and Smith, R. 1899 H., Analysis of Drainage Waters from Field A of the Hatch Experiment Stations: Report on General Work in the Chemical Lab. Massachusetts Agr. Coll., Hatch Exp. Sta., Ann. Report II (1898), pp. 139, 140.

(96) Grannetto, The Study of Sulfur as a Fertilizer: Bol. 1912 Quind. Soc. Agr. Ital., 17:14, pp. 425-29.

(97) Greaves, J. E., Azofication: Soil Sci., Vol. 6, pp. 173. 1918

(98) Greaves, J. E., The Effect of Soluble Salts on In1910 ' Soluble Phosphates: Jour. Biol. Chem., Vol. 7, No. 4, pp. 287-319.

(99) Greaves, J. E., and Carter, E. C., The Action of Some 1919 Common Soil Amendments: Soil Sci., Vol. 7, pp. 121-160.

(100) Greaves, J. E., and Carter, E. F., and Goldthorpe, H. 1919 C., Influence of Salts on the Nitric Nitrogen accumulation in the soil. Jour. Agr. Res., Vol. 16, pp. 107-135.

(101) Greaves, J. E., and Herst, C. F., Some Factors In1917 fluencing the Quantitative Determination of Nitric Nitrogen in the Soil: Soil Sci., Vol. $4: 3$, pp. 179-203; Ref. (200-203).

(102) Griffiths, A. B., A Treatise on Manures, 3936, London. 1889

(103) Grignard, Van Rijn, “Die Glykoside,” pp. 183-1.98.

(104) Guffroy, The Question' of Sulphate of Iron: Jour. 1907 Agr. Prat., n. s., t. 13, pp. 782-86.

(105) Hall, A*. D., Fertilizers and Manures, p. 186, London. 1909

(106) Hall, A. D., The Soil, an Introduction to the Scientific 1915 Study of the Growth of Crops, New York.

(107) Hall, A. D., The Soil. Quoted by Hart, E. B., and 1906 Peterson, W. H., Wisconsin Agr. Exp. Sta. Res. Bul. 14, p. 16. 
(108) Hall, A. D., The Soils, pp. 200-220. New York. 1906

(109) Hall, A. D., Some Secondary Actions of Manure on 1909 Soils: Jour. Royal Agr. 'Soc. England, 70, pp. 12-35.

(110) Hall, A. D., Binchley and Underwood, Soil Sol and Mineral Constituents of Soil: Jour. A'gr. Sci., Vol. 6:3, pp. 278-300.

(111) Hall, A. D., and Gimingham, Interaction of Ammonium 1907 Salts and the Constituents of the Soil: Trans. Chèm. Soc., 91, pp. 677.

(112) Halsted, B. D., New Jersey Report, 18, pp. 276-284. 1898

(113) Happen and Quensell, Transformation of Sulfur 1915 Compounds in Soil: Landw. Vers. Stat., 86, Nos. 1 and 2, pp. 1-24.

(114) Hart, E. B., and Peterson, W. H., Amer. Chem. Soc. 1911 Jour., Vol. 33, 549.

(115) Hart, E. B., and Peterson, W. H., Sulfur Requirements 1911 of Farm Crops in Relation to the Soil and Air Supply: Univ. of Wisconsin Agr. Exp. Sta. Res. Bul. 14.

(116) Hart, E. B., and Tottingham, W. E., Relation of Sulfur 1915 Compounds to Plant Nutrition: Jour. Agr. Res., Vol. 5, pp. 233-250.

(117) Harter, Bul. I, Va. Truck Exp. Sta. Rec.

(118) Hartwell, A. D., and Pember, F. R., The Presence of 1918 Aluminum as a Reason for the Difference in the Effect of the so-called Acid Soil on Barley and Rye: Soil Sci., Vol. 6, pp. 257-279.

(119) Hartwell, B. L., and Pember, F. R., The Toxicity af 1908 Ferrous Sulphate to Barley and Rye Seedlings: Rhode Island Jour. Exp. Sta. 21st Ann. Report, pp. 286-94.

(120) Heintz, B., Increasing the Productiveness of the Soil 1913 by Means of Sulfur: Naturwissenshaften, 1:5, 111-113; Exp. Sta. Rec. 28:8, p. 726.

(121) Herlinger, D., A Fertilization Experiment with Sulfur 
Applied to Potatoes: Wiener Landw. ZLtg., 62, 132; Chem. Abst., 6:9, p. 1201.

(122) Hilgard, E. W., Soils: Their Formation, Properties, 1906 Composition and Relation to Climate and Plant Growth in the Humid and Arid Regions, pp. 23, 208-209, New York.

(123) Hill, H. H., Potculture Experiments, 1904: Jour. 1905 Royal Agr. Soc. England, 66, p. 205.

(124) Hopkins, C. G., and Whiting, Albert L., Soil Bacteria 1916 and Phosphates: Illinois Agr. Exp. Sta. Bul. 190, pp. 391-406.

(125) Hopkins, C. G., Soil Fertility and Permanent Agricul1910 ture, pp. 75, New York.

(126) Hopkins, C. G., Sulfur Supply of Soil: Breeders' Gaz1911 ette, 60:2, pp. 51, 52.

(127) Hunt, T. F., Pennsylvania Exp. Sta. Report, pp. 80-92. 1908-09.

(128) Hunier, Brron, Farm Method; of Applying Land 1909 Flaster in Western Oregon and Western Washington: Circ. 22, Bur. Pl. Ind.. U. S. Dept. Agr.

(129) Jamison, T., Influence of Soluble and Insoluble Phos1879 phate as Manure for Turnips: Centbl. Agr. Chem., Vol. 8, pp. 652-56.

(130) Janicand, W., Has Sulfur a Direct Effect on Plants?: 1914 Jahrig., Vol. 18:3, pp. 29-32; Chem. Abst., Vol. $8: 14$, p. 2592.

(131) Johnson, Jour. Bio. Chem., Vol. 9, pp. 439-462. 1911

(132) Jones, Linus H., and Shive, John W., The Influence of 1921 Iron in the Forms of Ferric Phosphate and Ferrous Sulphate upon the Growth of Wheat in a Nutrient Solution: Soil Sci., Vol. 11:2, pp. 92-101.

(133) Kalayna, Tomio, The Availability of Phosphoric Acid 1905 in Bone Dust Modified by the Presence of Gypsum: Bul. Coll. Agr. Tokyo, Vol. 6, 353; Jour. Chem. Soc. Abst.; 88, pt. 2, p. 347.

(134) Kappen and Quenisell, Uber die Umwandlungen von 1915 Schwefel und Schwefelverbindungen im Ack- 
erboden, etc.: Landw. Vers. Stat., Vol. 86, pp. $1-34$.

(135) Kossovitch, Russ. Jour. Exp. Landw., 14, pp. 181-228; 1913 Chem. Abst., 8:5, p. 978.

(136) Krocker and Grahl, Manuring Experiments with Var1881 ious Phosphates: Landw. Vers. Stat. Bd. 27, pp. $151-160$.

(137) Laws and Gilbert, On the Composition of the Ash of 1884 Wheat Grain and Wheat Straw Grown at Rothamsted, in Different Seasons, and with Different Manures. Jour. Chem. Soc., London, $45,307$.

(138) Liechti, P., Action of Sulfur on Growth of Plants: Mitt. Lebensen Hyg., 4, 267; Chem. Abst., $8: 4$, p. 774 .

(139) Liercke, E., The Action of Sulfur in the Soil: Deut. 1913 Obstbau. Ztg., Vol. 59:4, pp. 75-76; Chem. Abst., $7: 16$, p. 2823.

(140) Lint, H. Clay, The Influence of Sulfur on Soil Acidity: 1914 Jour. Ind. and Engr. Chem., Vol. 6, p. 747.

(141) Lipman, Botan. Gazette, 51, 454. 1911

(142) Lipman, C. B., and Gericke, W. F., Does Calcium Car1918 bonate and Calcium Sulphate Treatment Affect the Solubility of the Soils' Constituents?: Univ. of Calif. Pub. in Agr. Sci. 3, pp. 271-282.

(143) Lipman, Chas. B., and Gericke, W. F., The Signif1918 cance of the Sulfur in Sulphate of Ammonia Applied to Certain Soils: Soil Sci., Vol. 5, pp. 81-86.

(144) Lipman, C. B., and Gericke, W. F., A Vegetation 1916 Experiment on the Availability of Nitrogenous Fertilizers in an Arid Soil: Soil Sci., Vol. 2:6, pp. 575-581.

(145) Lipman, C. B., and Sharp, L. T., New Experiments 1915 on Alkali Soil Treatment: Univ. Calif. Bul. Agr. Sci., Vol. 1, pp. 279-300.

(146) Lipman, J. G., Studies in Nitrification: Jour. Amer. 1902 Chem. Soc., Vol. 24, p. 171. 
(147) Lipman, J. G., Sulfur on Alkali Soils: Soil Sci., Vol. $1916 \quad 2: 8$, p. 205.

(148) Lipman, J. G., Report of New Jersey Agr. Exp. Sta., 1909 pp. 219-22.

(149) Lipman, J. G., Blair, McLean, H. C., and Wilkins., Fac1914 tors Influencing the Protein Conttent of Soy Bean: New Jersey Agr. Exp. Sta. Bul. 282, 250.

(150) Lipman, J. G., Blair, A. W., Martin, W. H., and Beck1921 with, C. S., Inoculated iSulfur as a PlantFood Solvent: Soil Sci., Vol. 11:2, pp. 87-93.

(151) Lipman, J. G., Blair, Owen, McLean, H. C., Influence 1912 of Ferrous Sulphate and Gypsum on Crop Yield and Nitrogen Recovery. New Jersey Exp. Sta. Report, pp. 270-277.

(152) Lipman, J. G., and Joffe, J. S., The 'Influence of 1920 Initial Reaction on the Oxidation of Sulfur and the Formation of Available Phosphate: Soil Sci., Vol. 10:4, pp. 327-332.

(153) Lipman, J. G., and McLean, H. C., Experiments witli 1918 Sulfur-Phosphate Composts conducted under Field Conditions. Soil Sci., Vol. 5:3, p. 243.

(154) Lipman, J. G., McLean, H. C., and Lint, H. Clay, The 1916 Oxidation of Sulfur in Soils as a Means of Increasing the Availability of Mineral Phosphates. Soil Sci., Vol. 1, No. 6, p. 533.

(155) Lipman, J. G., McLean, H. C., and Lint, H. Clay, Sul1916 fur Oxidation in Soils and its Effect on the Availability of. Mineral Phosphates. Soil Sci., Vol. $2: 6$, p. 499.

(156) Lipman, J. G., and McLean, H. C., Vegetation Experi1917 ments on the Availability of Treated Phosphates. Soil Sci., Vol. 4:4, p. 337.

(157) Loew, Uses of Sulfur and Chlorine: U. S. Dept. Agr. Div. Veg. Phys. and Path. Bul. 18.

(158) Lyon, T. L., and Bizzell, J. S., Lysimeter Experiments: 1918 Memoir 12, Cornell Univ. Agr. Exp. Sta.

(159) MacIntire, W. H., Willis, L. G., and Holding, W. S., The 1917 Divergent Effects of Lime and Magnesia upon 
the Conservation of Soil Sulphur. Soil Sci., Vol. 4:3, pp. 231-235.

(160) McCall, A. G., and Smith, A. M., Effect of Manure1920 Sulfur Composts upon the Availability of the Potassium of Greensand: Jour. Agr. Res., Vol. XIX:6, pp. 239-256.

(161) McConnell, Primrose, A Ruakura Experiment. Jour. 1913 Agr. New Zealand, Dept. Agr., Vol. 7:3, p. 253.

(162) McCool, M. M., and Miller, C. E., Effect of Calcium 1920 Sulfate on the Solubility of Soils: Joúr. Ágr. Res., Vol. XIX:2, pp. 47-54.

(163) McGeorge, W., The Effect of Fertilizers on the Physi1915 cal Properties of Hawaiian Soils: Hawaii Agr. Exp. Sta. Bul. 38.

(164) McLean, H. C., The Oxidation of Sulfur by Micro-or1918 ganisms in its Relation to the Availability of Phosphates: Soil Sci. Vol. 5:4, p. 251.

(165) McMiller, P. R., Calcium Sulphate on Potash: Jour. 1918 Agr. Res., Vol. 14, pp. 61-66.

(166) Magnien, Sulfur in the Cultivation of Turnips and 1913 Beets. Jour. Soc. Nat. Hort. France, $4: 14 \mathrm{~m}$, pp. $54-56$.

(167) Maizeres, Sulfur as a New Fertilizing Material: En1911 grais, 26:885; Chem. Abst., 5:23, p. 3872.

(168) Mares, H., Des Transformations que Subit le Soufre 1869 en Poudre (Fleur de Soufre et Soufre 'Triture) : Compt. Rend., 69, pp. 974-79.

(169) Martine, William H., The Relation of Sulfur to Soil 1920 Acidity and to the Control of Potato Scab: Soil Sei., Vol. 9:6, p. 393.

(170) Metz, Phillip, The Use of Gypsum in Agriculture as a Preservative and Fertilizer. Translated by Paul R. Rockwood and Published by the Southern Gypsum Company, North Holston, Virginia.

(171) Miller, H. G., The Relation of Sulphate to Plant Growth 1919 and Composition: Jour. Agr. Res., Vol. 17, pp. $87: 102$.

(172) Miller, N. H. J., The Amount and Composition of the 1906 Drainage Through Unmanured and Uneropped 
Land. Barnfield, Rothamsted. Jour. Agr. Sci., Vol. 1, pp. 377-399.

(173) Morse and Curry, Availability of Soil Potash in Clay 1909 and Clay Loam Soil: New Hampshire Exp. Sta. Bul. 142, pp. 39-58.

(174) Nedokuchaev, N., Influence of Calcium Nitrate and 1913 Ammonium Sulphate on Assimilation of Phosphoric Acid of Raw Phosphates: Russ. Jour. Expt. Landw., 14:1, pp. 1-21.

(174a) Nicolas, G., The Mechanism of the Fertilizing Action 1921 of Sulfur: Compt. Rend., 172, pp. 85-87.

(175) Niklewski, von Bronislaw, Conditions of Nitrifications 1910 in Stall Manure: Centbl. Bakt. (etc.) Abst., 2, Bd. 26, p. 388.

(176) Nolte, O., Gypsum as a Fertilizer: Jour. Landw., 65, 1917 pp. 67-73; Jour. Chem. Soc., 1i2:660, p. 624.

(177) Norton, J. H., Quantity and Composition of Drainage 1908 Water and a Comparison of 'Temperature, Evaporation and Rainfall: Amer. Chem. Soc. Jour., 30, pp. 1186-1190.

(178) Olive, E. W.,, Southern Gypsum Company Pàmphlet. 1919

(179) Osborne., Sulfur in Protein Bodies: Jour. Amer. Chem. 1902 Soc., 24, pp. 140-67.

(180) Paterson, J. W., and.Scott, P. R., Influence of Certain 1912 Soil Constitutents upon Nitrification: Jour. Dept. Agr., Victoria, Vol. 10, pp. 393-400.

(181) Peck, S. S., Lysimeter Experiments: Hawaiian Sugar 1911 Planters' Assn. Exp. Sta. Agr. and Chem. Ser., Bul. 37, pp. 1-38.

(182) Peck, S. S., Some Bio-Chemical Investigations of Ha1910 waiian soils: Hawaiian Sugar Planters' Sta. Bul. 34, pp. 1-39.

(183) Pember., Phosphorus and Potassium Requirements of 1917 Barley: Rhode Island Exp. Sta. Bul. 169, p. 48.

(184) Peterson, W. H., Forms of Sulfur in Plant Materials and Their Variation With the Soil Supply: Jour. Amer. Chem. Soc., 36, pp. 1290-1300. 
(185) Peterson, W. H., Sulfur in Plant Nutrition: Wisconsin 1914 Bul. 240, pp. 18, 19.

(186) Pfeffer, W. A Treatise upon the Metabolism and 1899 Sources of Energy of Plants, The Physiology of Plants, Vol. I, p. 132.

(187) Pfeiffer, T., and Blanck, E., Beitrag Zur Wirkung des 1913 Schwefels auf die Pflanzen-Production: Landiw. Vers. Stat., Bd. 83, pp. 359-384.

(188) Pfeiffer, T., and Simmermacker, W., Beitrag zur 1915 Wirkung des Schwefels auf die Pflanzen- Production: Fuhlings, Landw. Ztg., Bd. 64:9-10, pp. 243-55.

(189) Pitz, W. J., The Effect of Elemental Sulfur and of Cal1916 cium Sulphate on Plant Life: Jour. Agr. Res., 5, p. 771.

(189a) Powers, W. L., Sulfur in Relation to Soil: Reclam. 1920 Rec., Vol. 11, p. 28; Exp. Sta. Rec., 42, p. 525.

(190) Prescott, J. A., The Reaction between Dilute Acid and 1914 Soil Phosphates: Prac. Chem. Soc., Vol. 30, p. 137.

(191) Prichard, P., Action Nitrificante Comparee de Quel1884 ques Contences Naturellemente on Ajoutes dans les Terres Vegetales: Compt. Rend. Acad. Sci. (Paris.), t. 98, pp. 1288-1290.

(192) Reimer, F. C., Sulfur Fertilizers for Alfalfa: Pacific 1914 Rural Press, 87, 787.

(193) Reimer, F. C., Sulfur as a Fertilizer for Alfalfa: 1915 Monthly Bul. State Com. Hort. Sacramento, California, 4, pp. 405-408.

(194) Reimer, F. C., and Tartar, H. V., Sulfur as a Fertilizer 1919 for Alfalfa in Southern Oregon: Oregon Agr. Bul., 163.

(195) Rettger and Others, Amer. Jour. Physiol., 6, 450. 1902

(196) Robinson, W. O., The Inorganic Composition of Some 1914 Important American Soils: Soils Bul. 122, U. S. Dept. Agr.

(197) Robinson, W. O., Steinkonig, L. A., and Fry, W. J., 
1917 Variation in the Chemical Composition of Soils. U. S. Dept. Agr. Bul. 551, p. 16.

(198) Ruprecht, R. W., and Morse, F. W., The Cause of the 1917 Injurious Effect of Sulphate of Ammonia used as a Fertilizer: Massachusetts Agr. Exp. Sta. Bul., pp. 176-185.

(199) Russell, E. J., Soils and Manure, Cambridge, England. 1915

(200) Russell, E. J., and Richards, E. H., Changes in Ma1919 nure: Jour. Agr. Sci., Vol. 8, p. 495.

(201) Sabashnikov, V. V., Experiments on the Fertilizing 1912 Action of Sulfur: Exp. Sta. Rec., 28:8, p. 726.

(202) Schneidewind, W., To Rescue the Loss of Nitrogen in 1907 Barnyard Manure: Landw. Jahrb., Bd. 36:4, p. 569.

(202a) Shull, Chas. A., Sulfur as a Fertilizer: Science, Vol. 1920 52, pp. 376-8.

(203) Severin, S. A., Gypsum for Fixing Ammonia in Decom1903 posing Manure: Jour. Exp. Landw., Bd. 4:1, p. 88.

(204) Severin, S. A., Gips als Ammiakbindende Substuntz bie 1904 der Verottung des Stallmistes: Centbl. Bakt. Abst., 2, bd. 11, pp. 389-396, and pp. 422-457.

(205) Shedd, O. M., The Effect of Sulfur on Different Crops 1917 and Soils: Jour. of Agr. Res., Vol. 11:4, pp. 91-103.

(206) Shedd, O. M., The Effect of Oxidation of Sulfur in 1919 Soils on the Solubility of Rock Phosphate and on Nitrification: Jour. Agr. Res., 18, p. 329.

(207) Shedd, O. M., The Relation of Sulfur to Soil Fertility: 1914 Bul. 188, Kentucky Agr. Exp. Sta., pp. 593630.

(208) Shedd, O. M., Sulfur Content of Soil: Bul. 174, Ken1913 tucky Agr. Exp. Sta., pp. 269-306.

(209) Sherbakoff, C. D., Potato Scab and Sulfur Disinfec1914 tion: Cornell Bul. 350, pp. 709-743.

(210) Sherbakoff, C. D., The After Effects of Sulfur on Soil: 1915 Phytopathology, Vol. 5:4, pp. 219-22.

(211) Singh, Thakur Mahedeo, The Effect of Gypsum on 
1920 Bacterial Activities in Soils: Soil Sei., Vol. $9: 6$, p. 437.

(212) Snyder, H., Soils. The Composition of Native and Cul1893 tivated Soils and the Effects of Continuous Cultivation upon their Fertility: Minnesota Agr. Exp. Sta. Bul. 30.

(213) Soane, The Action of Gypsum as a Fertilizer: Staz. 1908 Spes. Agr. Ital., Vol. 41:8, pp. 473-500; Chem. Centbl., Vol. 2:19, pp. 16-27.

(214) Stewart, G. R., Mod. Soil Extract: Jour. Agr. Res., Vol. 12:6, pp. 311-368.

(215) Stewart, Robert, Sulfur in Relation to Soil Fertility: 1920 Univi. Tllinois Agr. Exp. Sta. Bul. 227.

(216) ' Stoklasa, J., The Losses of Nitrogen in Barnyard Ma1894 nure and Their Prevention: Oesterr, Ungar, Ztschr, Zucherindus, u. Landw. Bd. 23, pp. 525-536; Abst. in Chem. Centbl., Bd. 45, Abt. $2: 19$, p. 800 .

(217) Stone, R. W., Calcium Sulphate, Products, Preparation and Use. Tech. Paper 155 Bur. of Mines, Dept. of Interior.

(218) Stopr, Fred, Kong, J., Bohmer, C., Cosack, C., and Weig1884 mann, H., Ueber den Einfluss con Kochsalzund zenksufathaltigen wasser auf boden und planzen: Centbl. Agr. Chem., Jahrg. 13, pp. 76-87.

(219) Storer, F. H., Agriculture, Vol. I, pp. 347, New York. 1899

(220) Storer, F. H., Agriculture in Some Relations to Chemis1897 try, Chas. Scribner \& Sons, New York.

(221) Strest, J. P., The Reduction of Nitrates in the Pres1900 ance of Barnyard Manure: New Jers ${ }^{\circ}$ Agr. Exp. Station. 21st Ann. Report, p. 87.

(222) Suchting., Relative Effect of Chili Sulphate and Am1907 monium Sulphate: Jour. Landw. 55, pp. 1-46; Chem. Abst. 1:13, 1754.

(223) Swanson, C. O., Chemical Analysis of Some Kansas 1914 Soils: Kansas Agr. Exp. Sta. Bul. 199, p. 633. 
(224) Swanson, C. O., and Miller, R. W., Sulfur Content-Soil 1917 and Loss: Soil Sci., Vol. 3, pp. 129-148.

(225) Takeuchi, Gypsum as a Manure: Bul. Col. Agr. Tokyo 1908 Imp. Univ., Vol. 7:5, pp. 583-597.

(226) Thalau, Walter, Die Einwerkung von im Boden Defind1913 lichen Sulfiten, von Thiosulfat un Schwefel auf des Wachstum Pflanzen: Landw. Vers. Stat., 82, Heft. 3-4, pp. 161-209; Chem. Abst., 7,23 .

(227) Thompson, Amer. Chem. Soc. Jour., 35, p. 1628. 1913

(228) Thorne, C. E., Williams, C. G., and Ames, J. W., Raw 1916 Phosphate Rock as a Fertilizer: Ohio Bul. .305, pp. 248-277.

(229) Tottingham, W. E., The Sulfur Requirement of the Red 1918 Clover Plant: Jour. Bio. Chem., Vol. 36, pp. 429-438.

(230) Tottingham, W. E., and Hart, E. B., Sulfur and Sulfur 1921 Composts in Relation to Plant Nutrition: Soil Sci., Vol. 11:1, pp. 49-65.

(231) Tottingham, W. E. and Hoffman, C., Nature of Changes 1913 in Solubility and Availability of Phosphorus in Fermenting Mixtures: Wisconsin Agr. Exp. Sta. Res. Bul. 29.

(232) Tressler, D. K., Soluble Potash in Salt Solutions: Soil 1918 Sci., Vol. 6, pp. 237-57.

(233) Tritschler, Sulfur as a Fertilizer: Deut. Landw. Presse, 1913 40, 33, p. 405; Exp. Sta. Rec., 29, p. 319. .

(234) Troug, E., Factors Influencing the Availability of 1912 Rock 'Phosphates. Wisconsin Res.: Bul. '20, pp. 17-51.

(235) Urban, Action of Sulfur on the Growth of Sugar Beets: Z. Zuckerind, Bohmen, 37, 441. Chem. Abst., $7: 21$, p. 3685 .

(236) Van Bemmelen, Landw. Vers. Stat., 37, 284.

(237) Vercier, J., Experience sur L'action Fertilisante du Soufre: Prog. Agr. Vit., t. 58, pp. 695-97.

(238) Vermoral and Danthony, E., Sulfur and Iron Pyrites as 
1913 Fertilizers: Jourl. Agr. Prat., n. s. 26, No. 47, p. 651; Abst. Exp. Sta. Rec., 30, p. 627.

(239) Vermoral, V., and Danthony, E., Sulfur and Iron Py1914 rites as Fertilizers. Engrais, 28, pp. 1304-6. Chem. Abst., 8, 545 .

(240) Vermoral, V., Etude de L'action Fertilisante du Soufre 1914 sur la Vigne: Bul. Soc. Nat. Agr. France, t. 74, No. 1, pp. 48-51.

(241) Voelcker, Augustus, Field Experiments of Crude Ger1867 man Potash Salts and Common Salt on Mangolds. Jour. Royal Soc. England, s. 2, Vol. 3, pp. 86-91.

(242) Voelcker, J. A., Sulfur as a Fertilizer: Jour. Royal 1913 Agr. Soc. England, Vol. 5, 74, p. 419.

(243) Wagner, P., The Fertilizing Value and the Preservation 1897 of Barnyard Manure: Landw. Vers. Stat., Bd. 48 , No. 3-5, p. 360.

(244) Warrington and Richard, Trans. Chem. Soc., Vol. 47, p. 758. (From J. I. E. Ch., No. 2, p. 259.)

(245) Way, J. T., On the Power of Soils to Absorb Manure: 1850 Jour. Royal Agr. Soc., Vol. 11, pp. 313-379.

(246) Wheeler, Hartwell and Moore, (After Effects.) Rhode 1899 Island Agr. Exp. Sta., 12th Ann. Report, pp. 163-167.

(247) Wheeler, H. J., and Hartwell, B. L., et al., Concerning 1906 the Function of Sodium Salts: Rhode Island Agr. Exp. Sta., 19th Ann. Report, pp. 189-316.

(248) Wheeler, H. J., Hartwell, B. L., and Moore, N. C. L., 1899 Potato Scab: Rhode Island 12th Ann. Report, pp. 163-167.

(249) Wiegner, George, Xum Basenaustauch in Ded Acker1912 ende. Jour. Laṇdw., Vol. 60, pp. 110-197.

(250) Will and Korner, Ann., 125, p. 357.

(251) Wilson, J. K., Physiological Studies of Bacillus Radi1917 cicala of the Soybean and Factors Influencing Nodule Formation: New York (Cornell) Agr. Exp. Sta. Bul. 386.

(252) Wolff's Ash Analysis. 
(253) Wolf-Osterberg, (Method.) Biochem. Z., 29, pp. 429438.

(254) Zolla, D., L'Action Fertilisante du Soufre sur la 1915 Vigne: Rev. Gen. Sei., t. 26, pp. 120-121.

BIBLIOGRAPHY OF THE LITERATURE CONCERNING THE VALUE OF SULPHUR AS A FERTILIZER* BY COURTENAY DE KALB

(IN MANUFACTURERS RECORD, BALTIMIORE, DEC. 20, 1917.).

Effect of Sulphur Upon Plants: S. Bogdonov, Zhur. Russ. Fiz. Chim. Obsch., 31 (1899), No. 4, p. 471; Abs. in Bull. Soé Chim. Paris, 3, ser., 22, (1899), No. 23, p. 965; Exp. Sta. Rec., Vol. 11, 723.

Fertilizing Value of Sulphur Water: Deut. Landw. Presse, 36 (1909), No. 67, p. 717; E. S. R., Vol. 22-25.

Action of Sulphur As a Fertilizer: E. Chancrin and A. Desriot, Jour. Agr. Prat., N. ser., 21 (1911), No. 14, pp. 427-429; Abs. in Engrais, 26 (1911), No. 25, pp. 684, 685; Rev. Gen. Sci., 22 (1911), No. 10, pp. 392, 393; Deut. Landw. Presse, 37 (1910), No. 18, p. 204; E. S. R., Vol. 25-519.

Sulphur Requirements of Farm Crops: E. B. Hart and W. H. Peterson, Wisconsin Sta. Research Bul. 14, pp. 21; E. S. R. Vol. 25-519.

Action of Sulphur in Plants: E. Boullanger, Compt. Rend. Acad. Sci. (Paris), 154 (1912); E. S. R., Vol. 27, 27.

Sulphur As a Fertilizer: A. Heraud, Petite Rev. Agr. et Hort. 18 (1912), No. 419, pp. 112, 113; E. S. R., Vol. 27, 326.

Sulphur As a Fertilizer: Bernhard, Deut. Landw. Presse, 39 (1912), No. 23, p. 275; E. S. R., Vol. 27, 422.

Sulphur As a Fertilizer: L. Degrully, Prog. Agr. et Vit. (Ed. l'Est. Centre), 33 (1912), No. 11, pp. 321-324; E. S. R., Vol. $27,422$.

Sulphur As a Fertilizer. Abs. in International Inst. Agr. (Rome)., Bul. Bur. Agr. Intel. and Plant Diseases, 3 (1912), No. 5, pp. 1109-1111; E. S. R., Vol. 27, 422.

Sulphur As a Fertilizer: F. Giannetto, Bol. Guind. Soc. Agr. Ital., 17 (1912), No. 14, pp. 425-429; E. S. R., Vol. 27, 629.

* Since gypsum furnishes sulphate sulphur, and since other forms of sulphur must be transformed into sulphate sulphur before becoming arailable as plant food this bibliography deserves a place here: 
The Mechanism and Fertilizing Action of Sulphur: E. Boullanger, And. Dugardin, Compt. Rend. Acad. Sci. (Paris), 155 (1912), No. 4, pp. 327-329; Abs. in Rev. Sci. (Paris), 50 (1912), II, No. 5, pp. 156, 157 ; Rev. Vit. 38 (1912), No. 976, pp. 250-252; Chem. Zentbl. (1912), II, No. 16, pp. 1392, 1393; E. S. R., Vol. 27, 726 .

The Fertilizing Action of Sulphur: A. Demolon, Compt. Rend. Acad. Sci. (Paris), 154 (1912), No. 8, pp. 524-526; Abs. in Rev. Sci. (Paris), 50 (1912), I, No. 9, p. 285; E. S. R., Vol. 26, 819. Review of the Progress in the Use of Sulphur, Gypsum, and other Fertilizers: C. Guffroy, Vie Agr. et Rurale (1912), No. 10, pp. 233-240; E. S. R., Vol. 27, 128.

Experiments with the Fertilizing Action of Sulphur: V. V. Sebashnikov, Zuhr. Opytn, Agron. (Russ. Jour. Expt. Landw.), 13 (1912), No. 6, pp. 817-822, Fig. 1; Abs. in International Inst. Agr. (Rome) ; Bul. Agr. Intel. and Plant Diseases, 4 (1913), No. 3, pp. 381, 382; E. S. R., Vol. 28, 726.

Increasing the Productiveness of Soils by Means of Sulphur: B. Heinze, Naturwisenschaften, 1 (1913); E. S. R., Vol. 2s, 726.

Experiments in Use of Sulphur As a Fertilizer for Turnips: A. Magnien, Jour. Soc. Nat. Hort. France, 4 ser., 14; E. S. R., Vol. 28, 740 .

Experiments in Use of Sulphur As a Fertilizer for Beets: A Magnien, Jour. Soc. Nat. Hort. France, 4 ser., 14 (1913), Jan., pp. 55, 56; E. R. S., Vol. 28, 740.

Effect of Sulphur Supply on Plant Growth: W. E. Tottingham, Wisconsin Sta. Bul. 228, p. 26; E. S. R., Vol. 28, 815.

Action of Sulphur in the Soil: E. Lienke, Deut. Obstbau Ztg. (1913); No. 4, pp. 75-76; E. S. R., Vol. 28, 820.

The Effects of Sulphites, Thiosulphate and Sulphur in the Soil on Plant Growth; W. Thalan, Landw. Vers. Stat., 82 (1913), No. 3-4, pp. 161-209, Fig. 8; Abs. in Chem. Zenbbl. (1913), II, No. 10, pp. 985, 986; Ztchr. Angew, Chem., 26 (1913), No. 82, Referatenteil, p. 601; E. S. R., Vol. 29, 521.

The Fertilizing Action of Sulphur, Sulphuric Acid and Sulphùrous Acid: Compt. Rend. Acad. Sci. (Paris), 156 (1913), No. 9, pp. 725-728; E. S. R., Vol. 29, 25.

The Fertilizing Action of Sulphur, Sulphuric Acid and Sul- 
phurous Acid: A. Demolon, Sta. Agron. Aisne Bul. (1912), pp. 33-41; E. S. R., Vol. 29, 26.

Influence of Sulphur on Growth of Potatoes and Beets: E. Chancrin and A. Desriot, Jour. Agr. Prat., N. ser., 23 (1912), No. 12 , pp. $365-367$.

Experiments on the Fertilizing Action of Sulphur: A. Desriot, Jour. Agr. Prat., N. ser., 25 (1913), No. 12, pp. 364-365; E. S. R., Vol. 29, 215.

'The Requirement of Sulphur Fertilization by the Rice Plant: Alice R. Thompson, Hawaii Sta. Rpt. (1912), pp. 64-73; E. S. R., Vol. 29, 231, 232.

Sulphur As a Fertilizer: Fritschler, Deut. Landw. Presse 40 (1913), No. 33, p. 405; E. S. R., Vol. 29, 319.

The Use of Sulphur for Turnips and Beets: A. Magnien, Jour. Soc. Nat. Hort. France, 4, ser. 14 (1913), Jan., pp. 54-56; E. S. R., Vol. 30, 138.

Sulphur As an Indirect Fertilizer: H. von Feilitzen, K. Lantbr. Akad. Handbl. och Tidskr., 52 (1913), No. 2, pp. 120-130; E. S. R., Vol. 30, 139.

Action of Sulphur As a Fertilizer: M. A. Demolon, Sta. Agron. Aisne Bul. (1912), pp. 33-41; E. S. R., Vol. 30, 435.

The Direct Effect of Sulphur on Plants: W. Janicaud, Gartenwelt, 18 (1914), No. 3, pp. 29-32, Fig. 4; E. S. R., Vol. 30, 532. Sulphur As a Fertilizer: V. Vermoral and E. Danthony, Jour. Agr. Prat., N. ser., 26 (1913), No. 47, pp. 651-653; Bul. Soc. Nat. Agr. France, 73 (1913), No. 9, pp. 696-702; Engrais, 28 (1913), No. 47, pp. 1304-1306; Bul. Agr. Algerie et Tunesie, 19 (1913), No. 20, pp. 397-400; Abs. in Jour. Bd. Agr. (London), 20 (1914), No. 3, pp. 545, 546; International Inst. Agr. (Rome) (1914), No. 1, pp. 61-63; E. S. R., Vol. 30, 627.

Fertilizing Action of Sulphur on Grapes: V. Vermoral, Bul. Soc. Nat. Agr. France, 74 (1914), No. 1, pp. 38-51; E. S. R., Vol. 30,822 .

Influence of Sulphur on Growth of Sugar Beets: J. Urban, Ztschr. Zuckerindus. Bohmen., 37 (1913), No. 9, pp. 441-444; E. S. R., Vol. 30, 834.

Fertilizing Action of Sulphur: E. Boullanger, Vie. Agr. et Rurale, 2 (1913), No. 9, pp. 247, 248; E. S. R., Vol. 31, 31.

Experiments on the Use of Sulphur As a Fertilizer: J. A. 
Boelcker, Jour. Roy. Agr. Soc. England, 74 (1913), pp. 411422, pes. 5; E. S. R., Vol. 31, 218.

Action of Sulphur on Plant Production: T. Pfeiffer and E. Blanck, Landw. Vers. Stat., 83 (1914), No. 5-6, pp. 359-383, Fig. 1; Abs. in Ztschr. Angew. Chem., 27 (1914), No. 37, Referatenteil, p. 298; E. S. R., Vol. 31, 220.

Sulphur Fertilizer for Alfalfa: F. C. Reimer, Pacific Rural Press, 87 (1914), No. 26, p. 717; E. S. R., Vol. 31, 424.

Fertilizing Action of Sulphur on the Vine: J. Chauzit, Rev. Vit., 4 (1914), No. 1052, pp. 175-179, Fig. 2; E. S. R., Vol. 31, 442.

The Question of Sulphur in Agriculture: E. Miege, Rev. Sci. (Paris), 52 (1914), I, No. 25, pp. 778-784; E. S. R., Vol. 31, 623.

Sulphur in Plant Nutrition: Wisconsin Sta. Bul. 240 (1914). pp. 18, 19, Fig. 1; E. R. S., Vol. 31, 817.

Forms of Sulphur in Plant Materials, and Their Variation With the Soil Supply: W. H. Peterson, Jour. Amer. Chem. Soc., 36 (1914), No. 6, pp. 1290-1300; Abs. in Jour. Chem. Soc. (London), 106 (1914), No. 621, I, p. 914; E. S. R., Vol. 31, 817.

Relation of Sulphur to Soil Fertility: O, M. Shedd, Kentucky Sta. Bul. 188 (1914), pp. 595-630; E. S. R., Vol. 31, 724.

Transformation of Sulphur and Sulphur Compounds in Agricultural Soil: H. Kappen and E. Quensell, Landw. Vers. Stat., 86 (1915), No. 1-2, pp. 1-34; E. S. R., Vol. 33, 815.

Sulphur and Permanent Soil Fertility: P. E. Brown and E. H. Kellogg, Jour. Amer. Soc. Agron., 7 (1915), No. 3, pp. 97108; E. S. R., Vol. 34, 27.

Action of Sulphur on Plant Production: T. Pfeiffer and W. Simmermacher, Fuling's Landw. Ztg., 64 (1915), No. 9-10, pp. 243-255, Fig. 1; E. S. R., Vol. 34, 331.

Fertilizing Action of Sulphur on Vines: Rev. Gen. Sci.. 26 (1915), No. 4, pp. 120, 121; E. S. R., Vol. 34, 331. 


\section{ADDENDUM}

Page 478

Henderson, Junius,

Aurand, H. A.,

Page 482

Rowe, Jesse Perry,
COLORADO.

Gypsum, in The Foothill; Formations of North Centrai Colorado: Colorado Geological Survey, First Annual Report, 1908, p. 179 . Also in Bull. 19, p. 88.

Gypsum, in Mineral Deposits of the Western Slope: Colorado Geological Survey Bull. 22, p. 54.

MONTANA.

Gypsum Deposits, in Sorne Heonomic Geology of Montana: University of Montana Bull. 50, Geol. Ser. No. 3, pp. 31-39; 1908. 
